\title{
Existence and Exponential Stability of Equilibrium Point for Fuzzy BAM Neural Networks with Infinitely Distributed Delays and Impulses on Time Scales
}

\author{
Yongkun Li, Lijie Sun, and Li Yang \\ Department of Mathematics, Yunnan University, Kunming, Yunnan 650091, China \\ Correspondence should be addressed to Yongkun Li; yklie@ynu.edu.cn
}

Received 28 November 2013; Revised 6 February 2014; Accepted 10 February 2014; Published 1 April 2014

Academic Editor: Ray K. L. Su

Copyright (c) 2014 Yongkun Li et al. This is an open access article distributed under the Creative Commons Attribution License, which permits unrestricted use, distribution, and reproduction in any medium, provided the original work is properly cited.

By using the fixed point theorem and constructing a Lyapunov functional, we establish some sufficient conditions on the existence, uniqueness, and exponential stability of equilibrium point for a class of fuzzy BAM neural networks with infinitely distributed delays and impulses on time scales. We also present a numerical example to show the feasibility of obtained results. Our example also shows that the described time and continuous neural time networks have the same dynamic behaviours for the stability.

\section{Introduction}

The bidirectional associative memory (BAM) neural network models were first introduced by Kosko (see [1]). BAM neural network is a special class of recurrent neural networks that can store bipolar vector pairs. It is composed of neurons arranged in two layers, the $X$-layer and $Y$-layer. This class of networks possesses good applications prospects in areas of pattern recognition, signal and image process, and automatic control. Such applications heavily depend on the dynamical behaviors of neural networks. Thus, the analysis of the dynamical behaviors is a necessary step for practical design neural networks. In particular, many researchers have studied the dynamics of BAM neural networks with or without delays including stability and existence of periodic solutions or almost periodic solutions. For the results on BAM neural networks, the reader may see [2-11] and reference therein.

In mathematical modeling of real world problems, we will encounter some inconveniences, for example, the complexity and the uncertainty or vagueness. For the sake of taking vagueness into consideration, fuzzy theory is considered as a suitable method. Yang et al. proposed fuzzy cellular neural network, which integrates fuzzy logic into the structure of traditional cellular neural networks and maintains local connectedness among cells [12]. Fuzzy neural network has fuzzy logic between its template input and/or output besides the sum of product operation. Studies have been revealed that fuzzy neural network has its potential in imagine processing and pattern recognition and some results have reported on the stability and periodicity of fuzzy neural networks. Besides, in reality, time delays often occur due to finite switching speeds of the amplifiers and communication time and can destroy a stable network or cause sustained oscillations, bifurcation, or chaos. Hence, it is important to consider both the fuzzy logic and delay effect on dynamical behaviors of neural networks. There have been many results on the fuzzy neural networks with time delays [13-21]. For example, in [22], under the assumption that the activation function $f_{j}(u)$ is a second differentiable bounded function, $j=1,2, \ldots, n$, the authors proved the exponential stability and obtained the domain of robust attraction of the equilibrium point of the following interval fuzzy neural network:

$$
\begin{aligned}
x_{i}^{\prime}(t)= & -a_{i} x_{i}(t)+\sum_{j=1}^{n} b_{i j} f_{j}\left(x_{j}(t)\right) \\
& +\bigwedge_{j=1}^{n} \alpha_{i j} f_{j}\left(x_{j}(t)\right)+\bigwedge_{j=1}^{n} T_{i j} u_{j}
\end{aligned}
$$




$$
\begin{aligned}
& +\bigvee_{j=1}^{n} \beta_{i j} f_{j}\left(x_{j}(t)\right)+\bigvee_{j=1}^{n} S_{i j} u_{j} \\
& +\sum_{j=1}^{n} c_{i j} \mu_{j}+I_{i}, \quad i=1,2, \ldots, n .
\end{aligned}
$$

In fact, both continuous and discrete systems are very important in implementation and applications. To avoid the troublesomeness of studying the dynamical properties for continuous and discrete systems, respectively, it is meaningful to study that on time scales, which was initiated by Stefan Hilger in his Ph.D. thesis in order to unify continuous and discrete analysis. Lots of scholars have studied neural networks on time scales and obtained many good results [23-29]. For example, in [30], the authors considered the existence and global exponential stability of an equilibrium point for a class of fuzzy BAM neural networks with time-varying delays in leakage terms on time scales. Moreover, many systems also undergo abrupt changes at certain moments due to instantaneous perturbations, which lead to impulsive effects. Therefore, it is significant to study the dynamics of impulsive systems. For example, in [31], the authors studied the exponential stability of the following impulsive system on time scales:

$$
\begin{aligned}
x_{i}^{\Delta}(t)= & -a_{i} x_{i}(t)+\sum_{j=1}^{m} b_{j i} f_{j}\left(y_{j}(t)\right) \\
& +\sum_{j=1}^{m} b_{j i} f_{j}\left(y_{j}\left(t-\sigma_{j}\right)\right)+\bigwedge_{j=1}^{m} \alpha_{j i} f_{j}\left(y_{j}\left(t-\sigma_{j}\right)\right) \\
& +\bigvee_{j=1}^{m} \beta_{j i} f_{j}\left(y_{j}\left(t-\sigma_{j}\right)\right)+\bigwedge_{j=1}^{m} T_{j i} \mu_{j} \\
& +\bigvee_{j=1}^{m} H_{j i} \mu_{j}+I_{i}, \quad t \neq t_{k}, t \in \mathbb{T}^{+}, i=1,2, \ldots, n, \\
\Delta x_{i}\left(t_{k}\right) & =I_{k}\left(x_{i}\left(t_{k}\right)\right), \quad i=1,2, \ldots, n, k=1,2, \ldots,
\end{aligned}
$$

where $\mathbb{T}^{+}=\mathbb{T} \cap(0,+\infty)$ and $\mathbb{T}$ is a time scale, and in [32], authors studied a class of fuzzy BAM neural networks with finite distributed delays and impulses, by using fixed point theorem and differential inequality techniques; they established the existence and global exponential stability of unique equilibrium point to the networks.

However, to the best of our knowledge, few papers were published on the exponential stability of fuzzy BAM neural networks with distributed delays and impulses on time scales. Motivated by the above, in this paper, we study the following fuzzy BAM neural networks with infinitely distributed delays and impulses on time scales:

$$
\begin{gathered}
x_{i}^{\Delta}(t)=-a_{i} x_{i}(t)+\sum_{j=1}^{m} c_{j i} \int_{0}^{\infty} k_{j i}(s) f_{j}\left(y_{j}(t-s)\right) \Delta s \\
+\bigwedge_{j=1}^{m} \alpha_{j i} \int_{0}^{\infty} k_{j i}(s) f_{j}\left(y_{j}(t-s)\right) \Delta s
\end{gathered}
$$

$$
\begin{gathered}
\quad+\bigvee_{j=1}^{m} \beta_{j i} \int_{0}^{\infty} k_{j i}(s) f_{j}\left(y_{j}(t-s)\right) \Delta s \\
+\bigwedge_{j=1}^{m} T_{j i} \mu_{j}+\bigvee_{j=1}^{m} H_{j i} \mu_{j}+I_{i}, \\
\quad t \neq t_{k}, t \in \mathbb{T}^{+}, i=1,2, \ldots, n, \\
\Delta x_{i}\left(t_{k}\right)=P_{k}\left(x_{i}\left(t_{k}\right)\right), \quad i=1,2, \ldots, n, k=1,2, \ldots, \\
y_{j}^{\Delta}(t)=-b_{j} y_{j}(t)+\sum_{i=1}^{n} d_{i j} \int_{0}^{\infty} h_{i j}(s) g_{i}\left(x_{i}(t-s)\right) \Delta s \\
+\bigwedge_{i=1}^{n} p_{i j} \int_{0}^{\infty} h_{i j}(s) g_{i}\left(x_{i}(t-s)\right) \Delta s \\
+\bigvee_{i=1}^{n} q_{i j} \int_{0}^{\infty} h_{i j}(s) g_{i}\left(x_{i}(t-s)\right) \Delta s \\
+\bigwedge_{i=1}^{n} F_{i j} v_{i}+\bigvee_{i=1}^{n} G_{i j} v_{i}+J_{j}, \\
\\
t \neq t_{k}, t \in \mathbb{T}^{+}, j=1,2, \ldots, m, \\
\Delta y_{j}\left(t_{k}\right)=Q_{k}\left(y_{j}\left(t_{k}\right)\right), \quad j=1,2, \ldots, m, k=1,2, \ldots,
\end{gathered}
$$

where $\mathbb{T}$ is a time scale that is closed under addition; $n, m$ are the numbers of neurons in layers; $x_{i}(t)$ and $y_{j}(t)$ denote the activations of the $i$ th neuron and the $j$ th neuron at time $t ; a_{i}>0$ and $b_{j}>0$ represent the rate with which the $i$ th neuron and the $j$ th neuron will reset their potential to the resting state in isolation when they are disconnected from the network and the external inputs; $f_{j}, g_{i}$ are the input-output functions (the activation functions); $c_{j i}, d_{i j}$ are elements of feedback templates; $\alpha_{j i}, p_{i j}$ denote elements of fuzzy feedback MIN templates and $\beta_{j i}, q_{i j}$ are elements of fuzzy feedback MAX templates; $T_{j i}, F_{i j}$ are fuzzy feed-forward MIN templates and $H_{j i}, G_{i j}$ are fuzzy feed-forward MAX templates; $\mu_{j}, v_{i}$ denote the input of the $i$ th neuron and the $j$ th neuron; the delayed feedback $k_{j i}(s)$ and $h_{i j}(s)$ are real valued nonnegative continuous functions defined on $\mathbb{T}^{+}$with $\int_{0}^{\infty} k_{j i}(s) \Delta s \leq k_{j i}$ and $\int_{0}^{\infty} h_{i j}(s) \Delta s \leq h_{i j}$, where $k_{j i}$ and $h_{i j}$ are nonnegative constants; $I_{i}$, $J_{j}$ denote biases of the $i$ th neuron and the $j$ th neuron, $i=1,2, \ldots, n, j=1,2, \ldots, m ; \wedge$ and $\vee$ denote the fuzzy AND and fuzzy OR operations, respectively; the impulsive moments $t_{k}$ satisfy $0 \leq t_{0}<t_{1}<t_{2}<\cdots$ and $\lim _{k \rightarrow \infty} t_{k}=\infty$.

For $z=\left(x_{1}, x_{2}, \ldots, x_{n}, y_{1}, y_{2}, \ldots, y_{m}\right) \in \mathbb{R}^{n+m}$, we define the norm as $\|z\|=\sum_{i=1}^{n}\left|x_{i}\right|+\sum_{j=1}^{m}\left|y_{j}\right|$. For the sake of convenience, we denote the $\mathbb{T}$-interval $[a, b]_{\mathbb{T}}$ as $[a, b]_{\mathbb{T}}:=$ $\{t \in \mathbb{T} \mid a \leq t \leq b\}$. The initial condition of (3) is of the form

$$
\begin{aligned}
& x_{i}(s)=\varphi_{i}(s), \quad s \in(-\infty, 0]_{\mathbb{T}}, i=1,2, \ldots, n, \\
& y_{j}(s)=\psi_{j}(s), \quad s \in(-\infty, 0]_{\mathbb{T}}, j=1,2, \ldots, m,
\end{aligned}
$$

where $\varphi_{i}(\cdot), \psi_{j}(\cdot)$ denote positive real-valued continuous functions on $(-\infty, 0]_{\mathbb{T}}$. 

tions:

Throughout this paper, we make the following assump-

$$
\begin{aligned}
& \left(H_{1}\right) \text { for } t, s \in \mathbb{T}, t \pm s \in \mathbb{T} \text {; } \\
& \left(H_{2}\right) f_{j}, g_{i} \in C(\mathbb{R}, \mathbb{R}) \text { and there exist positive constants } L_{j}^{f}, \\
& L_{i}^{g} \text { such that }
\end{aligned}
$$$$
\begin{gathered}
\left|f_{j}(u)-f_{j}(v)\right| \leq L_{j}^{f}|u-v|, \\
\left|g_{i}(u)-g_{i}(v)\right| \leq L_{i}^{g}|u-v|,
\end{gathered}
$$

for all $u, v \in \mathbb{R}, i=1,2, \ldots, n, j=1,2, \ldots, m$;

$\left(H_{3}\right)$ there exists a positive constant $p$ such that for $i=$ $1,2, \ldots, n, j=1,2, \ldots, m$

$$
\begin{aligned}
& \int_{0}^{\infty} e_{p}(t+s, t) k_{j i}(s) \Delta s<+\infty \\
& \int_{0}^{\infty} e_{p}(t+s, t) h_{i j}(s) \Delta s<+\infty
\end{aligned}
$$

$\forall t \in \mathbb{T}$.

Remark 1. It is oblivious that if $\mathbb{T}=\mathbb{R}$ or $\mathbb{T}=\mathbb{Z}$, then $\left(H_{1}\right)$ holds and that (1) and (2) are all special cases of (3). Our methods used in this paper are different from those used in $[22,30-32]$.

The organization of the rest of this paper is as follows. In Section 2, we introduce some preliminary results which are needed in later sections. In Section 3, we establish some sufficient conditions for the existence and uniqueness of the equilibrium point of (3). In Section 4, we prove the equilibrium point of (3) is exponentially stable. In Section 5, we give an example to illustrate the feasibility of our results obtained in previous sections.

\section{Preliminaries}

In this section, we state some preliminary results.

Definition 2 (see [33]). Let $\mathbb{T}$ be a nonempty closed subset (time scale) of $\mathbb{R}$. The forward and backward jump operators $\sigma, \rho: \mathbb{T} \rightarrow \mathbb{T}$ and the graininess $\mu: \mathbb{T} \rightarrow \mathbb{R}_{+}$are defined, respectively, by

$$
\begin{aligned}
& \sigma(t)=\inf \{s \in \mathbb{T}: s>t\}, \\
& \rho(t)=\sup \{s \in \mathbb{T}: s<t\}, \\
& \mu(t)=\sigma(t)-t .
\end{aligned}
$$

Definition 3 (see [33]). A function $r: \mathbb{T} \rightarrow \mathbb{R}$ is called regressive if

$$
1+\mu(t) r(t) \neq 0,
$$

for all $t \in \mathbb{T}^{k}$. The set of all regressive and $r d$-continuous functions $r: \mathbb{T} \rightarrow \mathbb{R}$ will be denoted by $\mathscr{R}$. We define the set $\mathscr{R}^{+}=\{r \in \mathscr{R}: 1+\mu(t) r(t)>0, \forall t \in \mathbb{T}\}$.
Lemma 4 (see [33]). Assume that $p, q: \mathbb{T} \rightarrow \mathbb{R}$ are two regressive functions; then
(i) $e_{0}(t, s) \equiv 1$ and $e_{p}(t, t) \equiv 1$;
(ii) $e_{p}(t, s)=1 / e_{p}(s, t)=e_{\ominus p}(s, t)$;
(iii) $e_{p}(t, s) e_{p}(s, r)=e_{p}(t, r)$;
(iv) $\left(e_{p}(t, s)\right)^{\Delta}=p(t) e_{p}(t, s)$.

Definition 5 (see [33]). A function $F: \mathbb{T}^{k} \rightarrow \mathbb{R}$ is called a delta antiderivative of $f: \mathbb{T} \rightarrow \mathbb{R}$ provided $F^{\Delta}=f$ holds for all $t \in \mathbb{T}^{k}$. In this case we defined the integral of $f$ by

$$
\int_{a}^{t} f(s) \Delta s=F(t)-F(a), \quad t \in \mathbb{T},
$$

and we have the following formula:

$$
\int_{t}^{\sigma(t)} f(s) \Delta s=\mu(t) f(t), \quad t \in \mathbb{T}^{k} .
$$

Lemma 6 (see [33]). Let $f, g$ be $\Delta$-differentiable functions on $T$; then

(i) $\left(v_{1} f+v_{2} g\right)^{\Delta}=v_{1} f^{\Delta}+v_{2} g^{\Delta}$, for any constants $v_{1}, v_{2}$;

(ii) $(f g)^{\Delta}(t)=f^{\Delta}(t) g(t)+f(\sigma(t)) g^{\Delta}(t)=f(t) g^{\Delta}(t)+$ $f^{\Delta}(t) g(\sigma(t))$.

Lemma 7 (see [33]). Assume that $p(t) \geq 0$ for $t \geq s$; then $e_{p}(t, s) \geq 1$.

Lemma 8 (see [33]). Suppose that $p \in \mathscr{R}^{+}$; then

(i) $e_{p}(t, s)>0$, for all $t, s \in \mathbb{T}$;

(ii) if $p(t) \leq q(t)$ for all $t \geq s, t, s \in \mathbb{T}$, then $e_{p}(t, s) \leq$ $e_{q}(t, s)$ for all $t \geq s$.

Lemma 9 (see [33]). If $p \in \mathscr{R}$ and $a, b, c \in \mathbb{T}$, then

$$
\begin{gathered}
{\left[e_{p}(c, \cdot)\right]^{\Delta}=-p\left[e_{p}(c, \cdot)\right]^{\sigma},} \\
\int_{a}^{b} p(t) e_{p}(c, \sigma(t)) \Delta t=e_{p}(c, a)-e_{p}(c, b) .
\end{gathered}
$$

Lemma 10 (see [33]). Let $a \in \mathbb{T}^{k}, b \in \mathbb{T}$ and assume that $f: \mathbb{T} \times \mathbb{T}^{k} \rightarrow \mathbb{R}$ is continuous at $(t, t)$, where $t \in \mathbb{T}^{k}$ with $t>a$. Also assume that $f^{\Delta}(t, \cdot)$ is $r d$-continuous on $[a, \sigma(t)]$. Suppose that for each $\varepsilon>0$, there exists a neighborhood $U$ of $\tau \in[a, \sigma(t)]$ such that

$$
\begin{aligned}
& \left|f(\sigma(t), \tau)-f(s, \tau)-f^{\Delta}(t, \tau)(\sigma(t)-s)\right| \\
& \quad \leq \varepsilon|\sigma(t)-s|, \quad \forall s \in U,
\end{aligned}
$$

where $f^{\Delta}$ denotes the derivative of $f$ with respect to the first variable. Then

(i) $g(t):=\int_{a}^{t} f(t, \tau) \Delta \tau$ implies $g^{\Delta}(t):=\int_{a}^{t} f^{\Delta}(t, \tau) \Delta \tau+$ $f(\sigma(t), t)$;

(ii) $h(t):=\int_{t}^{b} f(t, \tau) \Delta \tau$ implies $h^{\Delta}(t):=\int_{t}^{b} f^{\Delta}(t, \tau) \Delta \tau-$ $f(\sigma(t), t)$. 
Definition 11 (see [33]). For each $t \in \mathbb{T}$, let $N$ be a neighborhood of $t$; then we define the generalized derivative (or Dini derivative), $D^{+} u^{\Delta}(t)$, to mean that, given $\epsilon>0$, there exists a right neighborhood $N(\epsilon) \subset N$ of $t$ such that

$$
\frac{u(\sigma(t))-u(s)}{\sigma(t)-s} \leq D^{+} u^{\Delta}(t)+\epsilon
$$

for each $s \in N(\epsilon), s>t$. In case $t$ is right-scattered and $u(t)$ is continuous at $t$, this reduces to

$$
D^{+} u^{\Delta}(t)=\frac{u(\sigma(t))-u(t)}{\sigma(t)-t} .
$$

Definition 12 (see [33]). If $a \in \mathbb{T}$, sup $\mathbb{T}=\infty$, and $f$ is rdcontinuous on $[a, \infty)$, then we define the improper integral by

$$
\int_{a}^{\infty} f(t) \Delta t=\lim _{b \rightarrow \infty} \int_{a}^{b} f(t) \Delta t,
$$

provided this limit exists, and we say that the improper integral converges in this case. If this limit does not exist, then we say that the improper integral diverges.

Definition 13 (see [33]). If $a \in \mathbb{T}$, inf $\mathbb{T}=-\infty$, and $f$ is rdcontinuous on $(-\infty, a)$, then we define the improper integral by

$$
\int_{-\infty}^{a} f(t) \Delta t=\lim _{b \rightarrow-\infty} \int_{b}^{a} f(t) \Delta t
$$

provided this limit exists, and we say that the improper integral converges in this case. If this limit does not exist, then we say that the improper integral diverges.

Lemma 14 (see [33]). Let $f, g, h \in C_{r d}\left([a, b]_{\mathbb{T}}, \mathbb{R}\right)$ and $1 / p+$ $1 / q=1$ with $p>1$; then

$$
\begin{aligned}
& \int_{a}^{b}|h(x)||f(x) g(x)| \Delta x \\
& \quad \leq\left(\int_{a}^{b}|h(x)||f(x)|^{p} \Delta x\right)^{1 / p}\left(\int_{a}^{b}|h(x)||g(x)|^{q} \Delta x\right)^{1 / q} .
\end{aligned}
$$

Definition 15. A point $z^{*}=\left(x_{1}^{*}, x_{2}^{*}, \ldots, x_{n}^{*}, y_{1}^{*}, y_{2}^{*}, \ldots, y_{m}^{*}\right)^{T} \in$ $\mathbb{R}^{n+m}$ is said to be an equilibrium point of (3) if $z(t)=z^{*}$ is a solution of (3).

Lemma 16 (see [19]). Let $f_{j}$ be defined on $\mathbb{R}, j=1,2, \ldots, m$. Then for any $a_{i j} \in \mathbb{R}, i=1,2, \ldots, n, j=1,2, \ldots, m$, we have the following estimations:

$$
\begin{aligned}
& \left|\bigwedge_{j=1}^{m} a_{i j} f_{j}\left(u_{j}\right)-\bigwedge_{j=1}^{m} a_{i j} f_{j}\left(v_{j}\right)\right| \leq \sum_{j=1}^{m}\left|a_{i j}\right|\left|f_{j}\left(u_{j}\right)-f_{j}\left(v_{j}\right)\right|, \\
& \left|\bigvee_{j=1}^{m} a_{i j} f_{j}\left(u_{j}\right)-\bigvee_{j=1}^{m} a_{i j} f_{j}\left(v_{j}\right)\right| \leq \sum_{j=1}^{m}\left|a_{i j}\right|\left|f_{j}\left(u_{j}\right)-f_{j}\left(v_{j}\right)\right|,
\end{aligned}
$$

where $u_{j}, v_{j} \in \mathbb{R}, j=1,2, \ldots, m$.
Definition 17. Let $z^{*}=\left(x_{1}^{*}, x_{2}^{*}, \ldots, x_{n}^{*}, y_{1}^{*}, y_{2}^{*}, \ldots, y_{m}^{*}\right)^{T}$ be an equilibrium point of (3). If there exists a positive constant $\lambda$ with $-\lambda \in \mathscr{R}^{+}$such that, for $t_{0} \in(-\infty, 0]_{\mathbb{T}}$, there exists $M>1$ such that for an arbitrary solution $z(t)=\left(x_{1}(t), x_{2}(t), \ldots, x_{n}(t), y_{1}(t), y_{2}(t), \ldots, y_{m}(t)\right)^{T}$ of $(3)$ with initial value $\phi(s)=\left(\varphi_{1}(s), \varphi_{2}(s), \ldots, \varphi_{n}(s), \psi_{1}(s), \psi_{2}(s)\right.$, $\left.\ldots, \psi_{m}(s)\right)^{T}$ satisfies

$$
\begin{array}{r}
\left|z(t)-z^{*}\right|_{1} \leq M\left\|\phi-z^{*}\right\| e_{\ominus \lambda}\left(t, t_{0}\right), \\
t \in\left[t_{0}, \infty\right)_{\mathbb{T}}, t \geq t_{0},
\end{array}
$$

where $\left|z(t)-z^{*}\right|_{1}=\sum_{i=1}^{n}\left|x_{i}(t)-x_{i}^{*}\right|^{2}+\sum_{j=1}^{m}\left|y_{j}(t)-y_{j}^{*}\right|^{2}$, $\left\|\phi-z^{*}\right\|=\sum_{i=1}^{n} \sup _{s \in(-\infty, 0]_{\mathbb{T}}}\left\{\left|\varphi_{i}(s)-x_{i}^{*}\right|^{2}+\right.$ $\left.\sum_{j=1}^{m} \sup _{s \in(-\infty, 0]_{\mathbb{T}}}\left\{\left|\psi_{j}(s)-y_{j}^{*}\right|^{2}\right\}\right\}$. Then the equilibrium point $z^{*}$ is said to be exponentially stable.

\section{Existence and Uniqueness of the Equilibrium Point}

In this section, we discuss the existence and uniqueness of the equilibrium point of (3).

Without loss of generality, we assume that the impulsive jump vectors $P$ and $Q$ satisfy

$$
\begin{aligned}
& P\left(x^{*}\right)=\left(P_{1}\left(x_{1}^{*}\right), P_{2}\left(x_{2}^{*}\right), \ldots, P_{n}\left(x_{n}^{*}\right)\right)^{T}=0, \\
& Q\left(y^{*}\right)=\left(Q_{1}\left(y_{1}^{*}\right), Q_{2}\left(y_{2}^{*}\right), \ldots, Q_{n}\left(y_{n}^{*}\right)\right)^{T}=0 .
\end{aligned}
$$

That is, if $\left(x_{1}^{*}, x_{2}^{*}, \ldots, x_{n}^{*}, y_{1}^{*}, y_{2}^{*}, \ldots, y_{m}^{*}\right)^{T}$ is an equilibrium point of the following nonimpulsive system

$$
\begin{aligned}
x_{i}^{\Delta}(t)= & -a_{i} x_{i}(t)+\sum_{j=1}^{m} c_{j i} \int_{0}^{\infty} k_{j i}(s) f_{j}\left(y_{j}(t-s)\right) \Delta s \\
& +\bigwedge_{j=1}^{m} \alpha_{j i} \int_{0}^{\infty} k_{j i}(s) f_{j}\left(y_{j}(t-s)\right) \Delta s \\
& +\bigvee_{j=1}^{m} \beta_{j i} \int_{0}^{\infty} k_{j i}(s) f_{j}\left(y_{j}(t-s)\right) \Delta s \\
& +\bigwedge_{j=1}^{m} T_{j i} \mu_{j}+\bigvee_{j=1}^{m} H_{j i} \mu_{j}+I_{i}, \\
y_{j}^{\Delta}(t)= & -b_{j} y_{j}(t)+\sum_{i=1}^{n} d_{i j} \int_{0}^{\infty} h_{i j}(s) g_{i}\left(x_{i}(t-s)\right) \Delta s \\
& +\bigwedge_{i=1}^{n} p_{i j} \int_{0}^{\infty} h_{i j}(s) g_{i}\left(x_{i}(t-s)\right) \Delta s \\
& +\bigvee_{i=1}^{n} q_{i j} \int_{0}^{\infty} h_{i j}(s) g_{i}\left(x_{i}(t-s)\right) \Delta s \\
& t, \ldots, \\
&
\end{aligned}
$$




$$
\begin{aligned}
& +\bigwedge_{i=1}^{n} F_{i j} \nu_{i}+\bigvee_{i=1}^{n} G_{i j} \nu_{i}+J_{j}, \\
& t \in \mathbb{T}^{+}, \quad j=1,2, \ldots, m
\end{aligned}
$$

then it is also the equilibrium point of impulsive system (3).

Theorem 18. Let $\left(H_{1}\right)$ and $\left(H_{2}\right)$ hold. Suppose further that $\left(H_{4}\right) \theta<1$, where

$$
\begin{array}{r}
\theta=\max \left\{\max _{1 \leq j \leq m}\left\{\sum_{i=1}^{n} a_{i}^{-1} L_{j}^{f} k_{j i}\left(\left|c_{j i}\right|+\left|\alpha_{j i}\right|+\left|\beta_{j i}\right|\right)\right\},\right. \\
\left.\max _{1 \leq i \leq n}\left\{\sum_{j=1}^{m} b_{j}^{-1} L_{i}^{g} h_{i j}\left(\left|d_{i j}\right|+\left|p_{i j}\right|+\left|q_{i j}\right|\right)\right\}\right\} ;
\end{array}
$$

then (3) has one unique equilibrium point.

Proof. If $z^{*}=\left(x_{1}^{*}, x_{2}^{*}, \ldots, x_{n}^{*}, y_{1}^{*}, y_{2}^{*}, \ldots, y_{m}^{*}\right)^{T}$ is an equilibrium point of (3), then we have

$$
\begin{aligned}
a_{i} x_{i}^{*}= & \sum_{j=1}^{m} c_{j i} \int_{0}^{\infty} k_{j i}(s) f_{j}\left(y_{j}^{*}\right) \Delta s \\
& +\bigwedge_{j=1}^{m} \alpha_{j i} \int_{0}^{\infty} k_{j i}(s) f_{j}\left(y_{j}^{*}\right) \Delta s \\
& +\bigvee_{j=1}^{m} \beta_{j i} \int_{0}^{\infty} k_{j i}(s) f_{j}\left(y_{j}^{*}\right) \Delta s \\
& +\bigwedge_{j=1}^{m} T_{j i} \mu_{j}+\bigvee_{j=1}^{m} H_{j i} \mu_{j}+I_{i}, \\
b_{j} y_{j}^{*}= & \sum_{i=1}^{n} d_{i j} \int_{0}^{\infty} h_{i j}(s) g_{i}\left(x_{i}^{*}\right) \Delta s \\
& +\bigwedge_{i=1}^{n} p_{i j} \int_{0}^{\infty} h_{i j}(s) g_{i}\left(x_{i}^{*}\right) \Delta s \\
& +\bigvee_{i=1}^{n} q_{i j} \int_{0}^{\infty} h_{i j}(s) g_{i}\left(x_{i}^{*}\right) \Delta s \\
& +\bigwedge_{i=1}^{n} F_{i j} \nu_{i}+\bigvee_{i=1}^{n} G_{i j} \nu_{i}+J_{j},
\end{aligned}
$$

where $i=1,2, \ldots, n, j=1,2, \ldots, m$. To finish the proof, it suffices to prove that (23) has a unique solution. Define a mapping $\Phi: \mathbb{R}^{n+m} \rightarrow \mathbb{R}^{n+m}$ as follows:

$$
\begin{gathered}
\Phi\left(x_{1}, x_{2}, \ldots, x_{n}, y_{1}, y_{2}, \ldots, y_{m}\right) \\
=\left(\Phi_{1}\left(x_{1}\right), \Phi_{2}\left(x_{2}\right), \ldots, \Phi_{n}\left(x_{n}\right),\right. \\
\left.\Phi_{n+1}\left(y_{1}\right), \ldots, \Phi_{n+m}\left(y_{m}\right)\right)^{T},
\end{gathered}
$$

where

$$
\begin{aligned}
\Phi_{i}\left(x_{i}\right)= & a_{i}^{-1}\left[\sum_{j=1}^{m} c_{j i} \int_{0}^{\infty} k_{j i}(s) f_{j}\left(y_{j}\right) \Delta s\right] \\
& +a_{i}^{-1}\left[\bigwedge_{j=1}^{m} \alpha_{j i} \int_{0}^{\infty} k_{j i}(s) f_{j}\left(y_{j}\right) \Delta s\right] \\
& +a_{i}^{-1}\left[\bigvee_{j=1}^{m} \beta_{j i} \int_{0}^{\infty} k_{j i}(s) f_{j}\left(y_{j}\right) \Delta s\right] \\
& +a_{i}^{-1}\left[\bigwedge_{j=1}^{m} T_{j i} \mu_{j}+\bigvee_{j=1}^{m} H_{j i} \mu_{j}+I_{i}\right],
\end{aligned}
$$

$$
\begin{aligned}
\Phi_{n+j}\left(y_{j}\right)= & b_{j}^{-1}\left[\sum_{i=1}^{n} d_{i j} \int_{0}^{\infty} h_{i j}(s) g_{i}\left(x_{i}\right) \Delta s\right] \\
& +b_{j}^{-1}\left[\bigwedge_{i=1}^{n} p_{i j} \int_{0}^{\infty} h_{i j}(s) g_{i}\left(x_{i}\right) \Delta s\right] \\
& +b_{j}^{-1}\left[\sum_{i=1}^{n} p_{i j} \int_{0}^{\infty} h_{i j}(s) g_{i}\left(x_{i}\right) \Delta s\right] \\
& +b_{j}^{-1}\left[\bigwedge_{i=1}^{n} F_{i j} \nu_{i}+\bigvee_{j=1}^{m} G_{i j} \nu_{i}+B_{j}\right],
\end{aligned}
$$

where $i=1,2, \ldots, n, j=1,2, \ldots, m$. Obviously, we need to show that $\Phi$ is a contraction mapping on $\mathbb{R}^{n+m}$. In fact, for any $\vartheta=\left(h_{1}, h_{2}, \ldots, h_{n}, v_{1}, v_{2}, \ldots, v_{m}\right)$ and $\bar{\vartheta}=$ $\left(\bar{h}_{1}, \bar{h}_{2}, \ldots, \bar{h}_{n}, \bar{v}_{1}, \bar{v}_{2}, \ldots, \bar{v}_{m}\right) \in \mathbb{R}^{n+m}$, we have

$$
\begin{gathered}
\left|\Phi_{i}\left(h_{i}\right)-\Phi_{i}\left(\bar{h}_{i}\right)\right| \\
=\mid a_{i}^{-1}\left[\sum_{j=1}^{m} c_{j i} \int_{0}^{\infty} k_{j i}(s)\left(f_{j}\left(v_{j}\right)-f_{j}\left(\bar{v}_{j}\right)\right) \Delta s\right] \\
+a_{i}^{-1}\left[\bigwedge_{j=1}^{m} \alpha_{j i} \int_{0}^{\infty} k_{j i}(s)\left(f_{j}\left(v_{j}\right)-f_{j}\left(\bar{v}_{j}\right)\right) \Delta s\right] \\
\quad+a_{i}^{-1}\left[\bigvee_{j=1}^{m} \beta_{j i} \int_{0}^{\infty} k_{j i}(s)\left(f_{j}\left(v_{j}\right)-f_{j}\left(\bar{v}_{j}\right)\right) \Delta s\right] \mid \\
\leq a_{i}^{-1} \sum_{j=1}^{m} L_{j}^{f} k_{j i}\left(\left|c_{j i}\right|+\left|\alpha_{j i}\right|+\left|\beta_{j i}\right|\right)\left|v_{j}-\bar{v}_{j}\right|, \\
i=1,2, \ldots, n,
\end{gathered}
$$




$$
\begin{gathered}
\left|\Phi_{n+j}\left(v_{j}\right)-\Phi_{n+j}\left(\bar{v}_{j}\right)\right| \\
=\mid b_{j}^{-1}\left[\sum_{i=1}^{n} d_{i j} \int_{0}^{\infty} h_{i j}(s)\left(g_{i}\left(h_{i}\right)-g_{i}\left(\bar{h}_{i}\right)\right) \Delta s\right] \\
+b_{j}^{-1}\left[\bigwedge_{i=1}^{n} p_{i j} \int_{0}^{\infty} h_{i j}(s)\left(g_{i}\left(h_{i}\right)-g_{i}\left(\bar{h}_{i}\right)\right) \Delta s\right] \\
+b_{j}^{-1}\left[\bigvee_{i=1}^{n} q_{i j} \int_{0}^{\infty} h_{i j}(s)\left(g_{i}\left(h_{i}\right)-g_{i}\left(\bar{h}_{i}\right)\right) \Delta s\right] \mid \\
\leq b_{j}^{-1} \sum_{i=1}^{n} L_{i}^{g} h_{i j}\left(\left|d_{i j}\right|+\left|p_{i j}\right|+\left|q_{i j}\right|\right)\left|h_{i}-\bar{h}_{i}\right|, \\
j=1,2, \ldots, m .
\end{gathered}
$$

Therefore, we have

$$
\begin{aligned}
& \|\Phi(\vartheta)-\Phi(\bar{\vartheta})\| \\
& =\sum_{i=1}^{n}\left|\Phi_{i}\left(h_{i}\right)-\Phi_{i}\left(\bar{h}_{i}\right)\right| \\
& +\sum_{j=1}^{m}\left|\Phi_{n+j}\left(v_{j}\right)-\Phi_{n+j}\left(\bar{v}_{j}\right)\right| \\
& \leq \sum_{i=1}^{n} a_{i}^{-1} \sum_{j=1}^{m} L_{j}^{f} k_{j i}\left(\left|c_{j i}\right|+\left|\alpha_{j i}\right|+\left|\beta_{j i}\right|\right)\left|v_{j}-\bar{v}_{j}\right| \\
& +\sum_{j=1}^{m} b_{j}^{-1} \sum_{i=1}^{n} L_{i}^{g} h_{i j}\left(\left|d_{i j}\right|+\left|p_{i j}\right|+\left|q_{i j}\right|\right)\left|h_{i}-\bar{h}_{i}\right| \\
& \leq \max _{1 \leq j \leq m}\left\{\sum_{i=1}^{n} a_{i}^{-1} L_{j}^{f} k_{j i}\left(\left|c_{j i}\right|+\left|\alpha_{j i}\right|+\left|\beta_{j i}\right|\right)\right\} \\
& \times \sum_{j=1}^{m}\left|v_{j}-\bar{v}_{j}\right| \\
& +\max _{1 \leq i \leq n}\left\{\sum_{j=1}^{m} a_{i}^{-1} L_{i}^{g} h_{i j}\left(\left|d_{i j}\right|+\left|p_{i j}\right|+\left|q_{i j}\right|\right)\right\} \\
& \times \sum_{i=1}^{n}\left|h_{i}-\bar{h}_{i}\right| \\
& \leq \theta\left(\sum_{i=1}^{n}\left|h_{i}-\bar{h}_{i}\right|+\sum_{j=1}^{m}\left|v_{j}-\bar{v}_{j}\right|\right) \\
& =\theta\|\vartheta-\bar{\vartheta}\| \text {. }
\end{aligned}
$$

By $\left(H_{3}\right)$, we obtain that $\Phi: \mathbb{R}^{n+m} \rightarrow \mathbb{R}^{n+m}$ is a contraction mapping. By the fixed point theorem of Banach space, there exists a unique fixed point of $\Phi$, which is a solution of (21).
Therefore, (3) has exactly one equilibrium point. The proof of Theorem 18 is completed.

\section{Exponential Stability of Equilibrium Point}

In this section, we consider the impulsive fuzzy BAM neural networks of the following type:

$$
\begin{aligned}
x_{i}^{\Delta}(t)= & -a_{i} x_{i}(t) \\
& +\sum_{j=1}^{m} c_{j i} \int_{0}^{\infty} k_{j i}(s) f_{j}\left(y_{j}(t-s)\right) \Delta s \\
& +\bigwedge_{j=1}^{m} \alpha_{j i} \int_{0}^{\infty} k_{j i}(s) f_{j}\left(y_{j}(t-s)\right) \Delta s \\
& +\bigvee_{j=1}^{m} \beta_{j i} \int_{0}^{\infty} k_{j i}(s) f_{j}\left(y_{j}(t-s)\right) \Delta s \\
& +\bigwedge_{j=1}^{m} T_{j i} \mu_{j}+\bigvee_{j=1}^{m} H_{j i} \mu_{j}+I_{i}, \\
\Delta x_{i}\left(t_{k}\right)=-\gamma_{i k} & \left(x_{i}\left(t_{k}\right)-x_{i}^{*}\right), \quad k=1,2, \ldots, i=1,2, \ldots, n, \\
y_{j}^{\Delta}(t)=- & b_{j} y_{j}(t)+\sum_{i=1}^{n} d_{i j} \int_{0}^{\infty} h_{i j}(s) g_{i}\left(x_{i}(t-s)\right) \Delta s \\
& +\bigwedge_{i=1}^{n} p_{i j} \int_{0}^{\infty} h_{i j}(s) g_{i}\left(x_{i}(t-s)\right) \Delta s \\
& +\bigvee_{i=1}^{n} q_{i j} \int_{0}^{\infty} h_{i j}(s) g_{i}\left(x_{i}(t-s)\right) \Delta s \\
& +\bigwedge_{i=1}^{n} F_{i j} v_{i}+\bigvee_{i=1}^{n} G_{i j} v_{i}+J_{j}, \\
& t \in \mathbb{T}^{+}, j=1,2, \ldots, m, \ldots, n, \\
& \Delta y_{j}\left(t_{k}\right)=-\bar{\gamma}_{j k}\left(y_{j}\left(t_{k}\right)-y_{j}^{*}\right), \\
& =1,2, \ldots, j=1,2, \ldots, m, \\
&
\end{aligned}
$$

where $a_{i}, b_{j}, c_{j i}, \beta_{j i}, \alpha_{j i}, d_{i j}, p_{i j}, q_{i j}, k_{j i}, h_{i j}, f_{j}, g_{i}$ are defined as those in (3). The initial conditions associated with (28) are given by (4). In the following, we study the exponential stability of the unique equilibrium point for (28) on time scales by using Lyapunov method.

Theorem 19. Let $\left(H_{1}\right)-\left(H_{4}\right)$ hold. Suppose further that $\left(H_{4}\right)$ there exists a constant $p>0$ such that

$$
\begin{aligned}
p & +(1+p \mu) \\
& \times\left(4 a_{i}^{2} \mu-2 a_{i}+\sum_{j=1}^{m} L_{j}^{f} k_{j i}\left(\left|c_{j i}\right|+\left|\alpha_{j i}\right|+\left|\beta_{j i}\right|\right)\right)
\end{aligned}
$$




$$
\begin{aligned}
& +\sum_{j=1}^{m} L_{i}^{g}(1+p \mu) \\
& \times\left(\left|d_{i j}\right|+\left|p_{i j}\right|+\left|q_{i j}\right|+4 n \mu L_{i}^{g} h_{i j}\left(d_{i j}^{2}+p_{i j}^{2}+q_{i j}^{2}\right)\right) \\
& \times \int_{0}^{\infty} e_{p}(t+s, t) h_{i j}(s) \Delta s<0, \quad i=1,2, \ldots, n, \\
p+ & (1+p \mu) \\
& \times\left(4 b_{j}^{2} \mu-2 b_{j}+\sum_{i=1}^{n} L_{i}^{g} h_{i j}\left(\left|d_{i j}\right|+\left|p_{i j}\right|+\left|q_{i j}\right|\right)\right) \\
& +\sum_{i=1}^{n} L_{j}^{f}(1+p \mu) \\
& \times\left(\left|c_{j i}\right|+\left|\alpha_{j i}\right|+\left|\beta_{j i}\right|+4 m \mu L_{j}^{f} k_{j i}\left(c_{j i}^{2}+\alpha_{j i}^{2}+\beta_{j i}^{2}\right)\right) \\
& \times \int_{0}^{\infty} e_{p}(t+s, t) k_{j i}(s) \Delta s<0, \quad j=1,2, \ldots, m,
\end{aligned}
$$

where $\mu=\sup _{t \in \mathbb{T}} \mu(t)<\infty$.

$\left(H_{5}\right) 0<\gamma_{i k}<2$ and $0<\bar{\gamma}_{j k}<2, i=1,2, \ldots, n, j=$ $1,2, \ldots, m, k=1,2, \ldots$.

Then the equilibrium point $z^{*}=\left(x_{1}^{*}, x_{2}^{*}, \ldots, x_{n}^{*}, y_{1}^{*}\right.$, $\left.y_{2}^{*}, \ldots, y_{m}^{*}\right)^{T}$ of $(28)$ is exponentially stable.

Proof. By Theorem 18, (28) has one unique equilibrium point $z^{*}=\left(x_{1}^{*}, x_{2}^{*}, \ldots, x_{n}^{*}, y_{1}^{*}, y_{2}^{*}, \ldots, y_{m}^{*}\right)^{T}$. Let $z(t)=\left(x_{1}(t), x_{2}(t)\right.$, $\left.\ldots, x_{n}(t), y_{1}(t), y_{2}(t), \ldots, y_{m}(t)\right)^{T}$ be an arbitrary solution of (28). Denote $u_{i}(t)=x_{i}(t)-x_{i}^{*}, v_{j}(t)=y_{j}(t)-y_{j}^{*}, i=$ $1,2, \ldots, n, j=1,2, \ldots, m$. Then from (28), we have the following:

$$
\begin{aligned}
& u_{i}^{\Delta}(t)=-a_{i} u_{i}(t) \\
&+\sum_{j=1}^{m} c_{j i} \int_{0}^{\infty} k_{j i}(s)\left(f_{j}\left(y_{j}(t-s)\right)-f_{j}\left(y_{j}^{*}\right)\right) \Delta s \\
&+\bigwedge_{j=1}^{m} \alpha_{j i} \int_{0}^{\infty} k_{j i}(s)\left(f_{j}\left(y_{j}(t-s)\right)-f_{j}\left(y_{j}^{*}\right)\right) \Delta s \\
&+\bigvee_{j=1}^{m} \beta_{j i} \int_{0}^{\infty} k_{j i}(s)\left(f_{j}\left(y_{j}(t-s)\right)-f_{j}\left(y_{j}^{*}\right)\right) \Delta s, \\
& t \in \mathbb{T}^{+}, i=1,2, \ldots, n,
\end{aligned}
$$$$
\Delta u_{i}\left(t_{k}\right)=-\gamma_{i k}\left(u_{i}\left(t_{k}\right)\right), \quad k=1,2, \ldots, i=1,2, \ldots, n,
$$$$
v_{j}^{\Delta}(t)=-b_{j} v_{j}(t)
$$$$
+\sum_{i=1}^{n} d_{i j} \int_{0}^{\infty} h_{i j}(s)\left(g_{i}\left(x_{i}(t-s)\right)-g_{i}\left(x_{i}^{*}\right)\right) \Delta s
$$$$
+\bigwedge_{i=1}^{n} p_{i j} \int_{0}^{\infty} h_{i j}(s)\left(g_{i}\left(x_{i}(t-s)\right)-g_{i}\left(x_{i}^{*}\right)\right) \Delta s
$$

$$
\begin{gathered}
+\bigvee_{i=1}^{n} q_{i j} \int_{0}^{\infty} h_{i j}(s)\left(g_{i}\left(x_{i}(t-s)\right)-g_{i}\left(x_{i}^{*}\right)\right) \Delta s, \\
t \in \mathbb{T}^{+}, j=1,2, \ldots, m, \\
\Delta v_{j}\left(t_{k}\right)=-\bar{\gamma}_{j k}\left(v_{j}\left(t_{k}\right)\right), \quad k=1,2, \ldots, j=1,2, \ldots, m .
\end{gathered}
$$

For $p>0$, construct Lyapunov functional $V(t)=V_{1}(t)+$ $V_{2}(t)+V_{3}(t)+V_{4}(t)$, where

$$
\begin{aligned}
V_{1}(t)= & \sum_{i=1}^{n} u_{i}^{2}(t) e_{p}(t, 0), \\
V_{2}(t)= & \sum_{j=1}^{m} v_{j}^{2}(t) e_{p}(t, 0), \\
V_{3}(t)=\sum_{i=1}^{n} \sum_{j=1}^{m} L_{j}^{f}(1+p \mu) & \\
& \times\left(\left|c_{j i}\right|+\left|\alpha_{j i}\right|+\left|\beta_{j i}\right|\right. \\
& \left.+4 m \mu L_{j}^{f} k_{j i}\left(c_{j i}^{2}+\alpha_{j i}^{2}+\beta_{j i}^{2}\right)\right) \\
& \times \int_{0}^{\infty} \int_{t-s}^{t} e_{p}(r+s, 0) k_{j i}(s) v_{j}^{2}(r) \Delta r \Delta s, \\
V_{4}(t)= & \sum_{j=1}^{m} \sum_{i=1}^{n} L_{i}^{g}(1+p \mu) \\
\times & \left(\left|d_{i j}\right|+\left|p_{i j}\right|+\left|q_{i j}\right|\right. \\
& \left.+4 n \mu L_{i}^{g} h_{i j}\left(d_{i j}^{2}+p_{i j}^{2}+q_{i j}^{2}\right)\right) \\
\times & \int_{0}^{\infty} \int_{t-s}^{t} e_{p}(r+s, 0) h_{j i}(s) u_{i}^{2}(r) \Delta r \Delta s .
\end{aligned}
$$

Calculating the $\Delta$-derivative $V^{\Delta}(t)$ of $V(t)$ along the solution of (30), we have

$$
\begin{aligned}
& V_{1}^{\Delta}(t)=\sum_{i=1}^{n}\left[e_{p}(\sigma(t), 0)\left(u_{i}^{2}(t)\right)^{\Delta}+u_{i}^{2}(t) e_{p}^{\Delta}(t, 0)\right] \\
& =\sum_{i=1}^{n}\left[e_{p}(\sigma(t), 0)\left(2 u_{i}(t) u_{i}^{\Delta}(t)+\mu(t)\left(u_{i}^{\Delta}(t)\right)^{2}\right)\right. \\
& \left.+p u_{i}^{2}(t) e_{p}(t, 0)\right] \\
& =\sum_{i=1}^{n}\left\{e_{p}(\sigma(t), 0)\right. \\
& \times\left[2 u_{i}(t)\right. \\
& \times\left(-a_{i} u_{i}(t)\right. \\
& +\sum_{j=1}^{m} c_{j i} \int_{0}^{\infty} k_{j i}(s)
\end{aligned}
$$




$$
\begin{aligned}
& \times\left(f_{j}\left(y_{j}(t-s)\right)-f_{j}\left(y_{j}^{*}\right)\right) \Delta s \\
& +\bigwedge_{j=1}^{m} \alpha_{j i} \int_{0}^{\infty} k_{j i}(s) \\
& \times\left(f_{j}\left(y_{j}(t-s)\right)-f_{j}\left(y_{j}^{*}\right)\right) \Delta s \\
& +\bigvee_{j=1}^{m} \beta_{j i} \int_{0}^{\infty} k_{j i}(s)\left(f_{j}\left(y_{j}(t-s)\right)\right. \\
& \left.\left.-f_{j}\left(y_{j}^{*}\right)\right) \Delta s\right) \\
& +\mu(t)\left(-a_{i} u_{i}(t)+\sum_{j=1}^{m} c_{j i}\right. \\
& \times \int_{0}^{\infty} k_{j i}(s) \\
& \times\left(f_{j}\left(y_{j}(t-s)\right)-f_{j}\left(y_{j}^{*}\right)\right) \Delta s \\
& +\bigwedge_{j=1}^{m} \alpha_{j i} \\
& \times \int_{0}^{\infty} k_{j i}(s) \\
& \times\left(f_{j}\left(y_{j}(t-s)\right)\right. \\
& \left.-f_{j}\left(y_{j}^{*}\right)\right) \Delta s \\
& +\bigvee_{j=1}^{m} \beta_{j i} \\
& \times \int_{0}^{\infty} k_{j i}(s) \\
& \times\left(f_{j}\left(y_{j}(t-s)\right)\right. \\
& \left.\left.\left.-f_{j}\left(y_{j}^{*}\right)\right) \Delta s\right)^{2}\right] \\
& \left.+p u_{i}^{2}(t) e_{p}(t, 0)\right\} \\
& \leq \sum_{i=1}^{n}\left\{p e_{p}(t, 0) u_{i}^{2}(t)+e_{p}(\sigma(t), 0)\right. \\
& \times\left[-2 a_{i} u_{i}^{2}(t)\right. \\
& +2 \sum_{j=1}^{m}\left(\left|c_{j i}\right|+\left|\alpha_{j i}\right|+\left|\beta_{j i}\right|\right) L_{j}^{f} \\
& \times \int_{0}^{\infty} k_{j i}(s)\left|v_{j}(t-s)\right|\left|u_{i}(t)\right| \Delta s \\
& +\mu(t)\left(4 a_{i}^{2} u_{i}^{2}(t)\right. \\
& +4\left(\sum_{j=1}^{m}\left|c_{j i}\right| L_{j}^{f}\right.
\end{aligned}
$$$$
\left.\times \int_{0}^{\infty} k_{j i}(s)\left|v_{j}(t-s)\right| \Delta s\right)^{2}
$$$$
+4\left(\sum_{j=1}^{m}\left|\alpha_{j i}\right| L_{j}^{f}\right.
$$$$
\left.\times \int_{0}^{\infty} k_{j i}(s)\left|v_{j}(t-s)\right| \Delta s\right)^{2}
$$$$
+4\left(\sum_{j=1}^{m}\left|\beta_{j i}\right| L_{j}^{f}\right.
$$$$
\times \int_{0}^{\infty} k_{j i}(s)
$$$$
\left.\left.\left.\left.\times\left|v_{j}(t-s)\right| \Delta s\right)^{2}\right)\right]\right\}
$$$$
\leq \sum_{i=1}^{n}\left\{p e_{p}(t, 0) u_{i}^{2}(t)+e_{p}(\sigma(t), 0)\right.
$$$$
\times\left[-2 a_{i} u_{i}^{2}(t)+\sum_{j=1}^{m}\left(\left|c_{j i}\right|+\left|\alpha_{j i}\right|+\left|\beta_{j i}\right|\right) L_{j}^{f}\right.
$$$$
\times \int_{0}^{\infty} k_{j i}(s)\left(v_{j}^{2}(t-s)+u_{i}^{2}(t)\right) \Delta s
$$$$
+\mu(t)\left(4 a_{i}^{2} u_{i}^{2}(t)+4 m \sum_{j=1}^{m} c_{j i}^{2}\left(L_{j}^{f}\right)^{2}\right.
$$$$
\times\left(\int_{0}^{\infty} k_{j i}(s)\left|v_{j}(t-s)\right| \Delta s\right)^{2}
$$$$
+4 m \sum_{j=1}^{m} \alpha_{j i}^{2}\left(L_{j}^{f}\right)^{2}
$$$$
\times\left(\int_{0}^{\infty} k_{j i}(s)\left|v_{j}(t-s)\right| \Delta s\right)^{2}
$$$$
+4 m \sum_{j=1}^{m} \beta_{j i}^{2}\left(L_{j}^{f}\right)^{2}
$$$$
\left.\left.\left.\times\left(\int_{0}^{\infty} k_{j i}(s)\left|v_{j}(t-s)\right| \Delta s\right)^{2}\right)\right]\right\}
$$$$
\leq \sum_{i=1}^{n}\left\{p e_{p}(t, 0) u_{i}^{2}(t)+e_{p}(\sigma(t), 0)\right.
$$$$
\times\left[-2 a_{i} u_{i}^{2}(t)\right.
$$ 
Journal of Applied Mathematics

9

$$
\begin{aligned}
& +\sum_{j=1}^{m}\left(\left|c_{j i}\right|+\left|\alpha_{j i}\right|+\left|\beta_{j i}\right|\right) L_{j}^{f} \\
& +\sum_{j=1}^{m} L_{j}^{f} k_{j i} \\
& \times \int_{0}^{\infty} k_{j i}(s)\left(v_{j}^{2}(t-s)+u_{i}^{2}(t)\right) \Delta s+\mu(t) \\
& \times\left(4 a_{i}^{2} u_{i}^{2}(t)\right. \\
& \left.\left.\times\left(\left|c_{j i}\right|+\left|\alpha_{j i}\right|+\left|\beta_{j i}\right|\right)\right]\right\} \\
& +4 m \sum_{j=1}^{m} c_{j i}^{2}\left(L_{j}^{f}\right)^{2} \int_{0}^{\infty} k_{j i}(s) \Delta s \\
& \times u_{i}^{2}(t)+e_{p}(t, 0) \sum_{i=1}^{n} \sum_{j=1}^{m}(1+p \mu(t)) \\
& \times \int_{0}^{\infty} k_{j i}(s) v_{j}^{2}(t-s) \Delta s \\
& \times\left[L_{j}^{f}\left(\left|c_{j i}\right|+\left|\alpha_{j i}\right|+\left|\beta_{j i}\right|\right)\right. \\
& +4 m \sum_{j=1}^{m} \alpha_{j i}^{2}\left(L_{j}^{f}\right)^{2} \int_{0}^{\infty} k_{j i}(s) \Delta s \\
& \times \int_{0}^{\infty} k_{j i}(s) v_{j}^{2}(t-s) \Delta s \\
& +4 m \sum_{j=1}^{m} \beta_{j i}^{2}\left(L_{j}^{f}\right)^{2} \\
& \times \int_{0}^{\infty} k_{j i}(s) \Delta s \\
& \left.\left.\left.\times \int_{0}^{\infty} k_{j i}(s) v_{j}^{2}(t-s) \Delta s\right)\right]\right\} \\
& \leq \sum_{i=1}^{n}\left\{p e_{p}(t, 0) u_{i}^{2}(t)+(1+p \mu(t)) e_{p}(t, 0)\right. \\
& \times\left[-2 a_{i} u_{i}^{2}(t)\right. \\
& +\sum_{j=1}^{m}\left(\left|c_{j i}\right|+\left|\alpha_{j i}\right|+\left|\beta_{j i}\right|\right) L_{j}^{f} k_{j i} u_{i}^{2}(t) \\
& +\sum_{j=1}^{m}\left(\left|c_{j i}\right|+\left|\alpha_{j i}\right|+\left|\beta_{j i}\right|\right) L_{j}^{f} \\
& \times \int_{0}^{\infty} k_{j i}(s) v_{j}^{2}(t-s) \Delta s \\
& +4 a_{i}^{2} \mu(t) u_{i}^{2}(t)+4 m \mu(t) \\
& \left.+4 m \mu(t)\left(L_{j}^{f}\right)^{2} k_{j i}\left(c_{j i}^{2}+\alpha_{j i}^{2}+\beta_{j i}^{2}\right)\right] \\
& \times \int_{0}^{\infty} k_{j i}(s) v_{j}^{2}(t-s) \Delta s \\
& \leq \sum_{i=1}^{n} e_{p}(t, 0) \\
& \times\{p+(1+p \mu) \\
& \times\left[4 a_{i}^{2} \mu-2 a_{i}\right. \\
& \left.\left.+\sum_{j=1}^{m} L_{j}^{f} k_{j i}\left(\left|c_{j i}\right|+\left|\alpha_{j i}\right|+\left|\beta_{j i}\right|\right)\right]\right\} \\
& \times u_{i}^{2}(t)+e_{p}(t, 0) \sum_{i=1}^{n} \sum_{j=1}^{m}(1+p \mu) \\
& \times\left\{\left[L_{j}^{f}\left(\left|c_{j i}\right|+\left|\alpha_{j i}\right|+\left|\beta_{j i}\right|\right)+4 m \mu\left(L_{j}^{f}\right)^{2}\right.\right. \\
& \left.\times k_{j i}\left(c_{j i}^{2}+\alpha_{j i}^{2}+\beta_{j i}^{2}\right)\right] \\
& \left.\times \int_{0}^{\infty} k_{j i}(s) v_{j}^{2}(t-s) \Delta s\right\} .
\end{aligned}
$$$$
\times \sum_{j=1}^{m}\left(c_{j i}^{2}+\alpha_{j i}^{2}+\beta_{j i}^{2}\right)\left(L_{j}^{f}\right)^{2} k_{j i}
$$$$
\left.\left.\times \int_{0}^{\infty} k_{j i}(s) v_{j}^{2}(t-s) \Delta s\right]\right\}
$$$$
=\sum_{i=1}^{n} e_{p}(t, 0)
$$$$
\times\{p+(1+p \mu(t))
$$$$
\times\left[4 a_{i}^{2} \mu(t)-2 a_{i}\right.
$$

Similarly, we have that

$$
\begin{aligned}
& V_{2}^{\Delta}(t)= \sum_{j=1}^{m}\left[e_{p}(\sigma(t), 0)\left(v_{j}^{2}(t)\right)^{\Delta}+v_{j}^{2}(t) e_{p}^{\Delta}(t, 0)\right] \\
&=\sum_{j=1}^{m}\left[e_{p}(\sigma(t), 0)\left(2 v_{j}(t) v_{j}^{\Delta}(t)+\mu(t)\left(v_{j}^{\Delta}(t)\right)^{2}\right)\right. \\
&\left.\quad+p v_{i}^{2}(t) e_{p}(t, 0)\right] \\
&=\sum_{j=1}^{m} e_{p}(\sigma(t), 0)
\end{aligned}
$$


10

Journal of Applied Mathematics

$$
\begin{aligned}
& \times\left[2 v_{i}(t)\right. \\
& \times v_{j}^{2}(t)+e_{p}(t, 0) \\
& \times \sum_{j=1}^{m} \sum_{i=1}^{n}(1+p \mu(t)) \\
& \times\left(-b_{j} v_{i}(t)\right. \\
& \times\left[L_{i}^{g}\left(\left|d_{i j}\right|+\left|p_{i j}\right|+\left|q_{i j}\right|\right)\right. \\
& +\sum_{j=1}^{m} d_{i j} \int_{0}^{\infty} h_{i j}(s) \\
& \left.+4 n \mu(t)\left(L_{i}^{g}\right)^{2}\left(d_{i j}^{2}+p_{i j}^{2}+q_{i j}^{2}\right)\right] \\
& \times\left(g_{i}\left(x_{i}(t-s)\right)-g_{i}\left(x_{i}^{*}\right)\right) \Delta s \\
& \times \int_{0}^{\infty} h_{i j}(s) u_{i}^{2}(t-s) \Delta s \\
& +\bigwedge_{i=1}^{n} p_{i j} \\
& \leq \sum_{j=1}^{m} e_{p}(t, 0)\{p+(1+p \mu) \\
& \times \int_{0}^{\infty} h_{i j}(s)\left(g_{i}\left(x_{i}(t-s)\right)-g_{i}\left(x_{i}^{*}\right)\right) \Delta s \\
& +\bigvee_{i=1}^{n} \beta_{i j} \int_{0}^{\infty} h_{i j}(s)\left(g_{i}\left(x_{i}(t-s)\right)\right. \\
& \times\left[4 b_{j}^{2} \mu-2 b_{j}\right. \\
& \left.\left.+\sum_{i=1}^{n} L_{i}^{g} h_{i j}\left(\left|d_{i j}\right|+\left|p_{i j}\right|+\left|q_{i j}\right|\right)\right]\right\} \\
& \times v_{j}^{2}(t)+e_{p}(t, 0) \\
& +\mu(t) \\
& \times \sum_{j=1}^{m} \sum_{i=1}^{n}(1+p \mu) \\
& \times\left(-b_{j} v_{j}(t)+\sum_{i=1}^{n} d_{i j}\right. \\
& \times\left[L_{i}^{g}\left(\left|d_{i j}\right|+\left|p_{i j}\right|+\left|q_{i j}\right|\right)\right. \\
& \times \int_{0}^{\infty} h_{i j}(s)\left(g_{i}\left(x_{i}(t-s)\right)-g_{i}\left(x_{i}^{*}\right)\right) \Delta s \\
& \left.+4 n \mu\left(L_{i}^{g}\right)^{2} h_{i j}\left(d_{i j}^{2}+p_{i j}^{2}+q_{i j}^{2}\right)\right] \\
& +\bigwedge_{i=1}^{n} p_{i j} \int_{0}^{\infty} h_{i j}(s) \\
& \times \int_{0}^{\infty} h_{i j}(s) u_{i}^{2}(t-s) \Delta s .
\end{aligned}
$$$$
\times\left(g_{i}\left(x_{i}(t-s)\right)-g_{i}\left(x_{i}^{*}\right)\right) \Delta s
$$

Besides, we can obtain that

$$
\begin{aligned}
V_{3}^{\Delta}(t)= & \sum_{i=1}^{n} \sum_{j=1}^{m} L_{j}^{f}(1+p \mu) \\
& \times\left(\left|c_{j i}\right|+\left|\alpha_{j i}\right|+\left|\beta_{j i}\right|\right. \\
& \left.\quad+4 m \mu L_{j}^{f} k_{j i}\left(c_{j i}^{2}+\alpha_{j i}^{2}+\beta_{j i}^{2}\right)\right) \\
& \times \int_{0}^{\infty} e_{p}(t+s, 0) k_{j i}(s) v_{j}^{2}(t) \Delta s \\
& -\sum_{i=1}^{n} \sum_{j=1}^{m} L_{j}^{f}(1+p \mu) \\
& \times\left(\left|c_{j i}\right|+\left|\alpha_{j i}\right|+\left|\beta_{j i}\right|\right. \\
& \times \int_{0}^{\infty} e_{p}(t, 0) k_{j i}(s) v_{j}^{2}(t-s) \Delta s
\end{aligned}
$$$$
+p v_{j}^{2}(t) e_{p}(t, 0)
$$$$
\left.\left.+\sum_{i=1}^{n} L_{i}^{g} h_{i j}\left(\left|d_{i j}\right|+\left|p_{i j}\right|+\left|q_{i j}\right|\right)\right]\right\}
$$ 
Journal of Applied Mathematics

11

$$
\begin{aligned}
& =\sum_{i=1}^{n} \sum_{j=1}^{m} L_{j}^{f}(1+p \mu) \\
& \times\left(\left|c_{j i}\right|+\left|\alpha_{j i}\right|+\left|\beta_{j i}\right|\right. \\
& \left.+4 m \mu L_{j}^{f} k_{j i}\left(c_{j i}^{2}+\alpha_{j i}^{2}+\beta_{j i}^{2}\right)\right) \\
& \times e_{p}(t, 0) \int_{0}^{\infty} e_{p}(t+s, t) k_{j i}(s) \Delta s v_{j}^{2}(t) \\
& -e_{p}(t, 0)(1+p \mu) \\
& \times \sum_{i=1}^{n} \sum_{j=1}^{m} L_{j}^{f}\left(\left|c_{j i}\right|+\left|\alpha_{j i}\right|+\left|\beta_{j i}\right|\right. \\
& \left.+4 m L_{j}^{f} k_{j i}\left(c_{j i}^{2}+\alpha_{j i}^{2}+\beta_{j i}^{2}\right)\right) \\
& \times \int_{0}^{\infty} k_{j i}(s) v_{j}^{2}(t-s) \Delta s, \\
& V^{\Delta}(t)=V_{1}^{\Delta}(t)+V_{2}^{\Delta}(t)+V_{3}^{\Delta}(t)+V_{4}^{\Delta}(t) \\
& \leq \sum_{i=1}^{n} e_{p}(t, 0) \\
& \times\{p+(1+p \mu) \\
& \times\left[4 a_{i}^{2} \mu-2 a_{i}\right. \\
& V_{4}^{\Delta}(t)=\sum_{j=1}^{m} \sum_{i=1}^{n} L_{i}^{g}(1+p \mu) \\
& +\sum_{j=1}^{m} L_{j}^{f} k_{j i} \\
& \times\left(\left|d_{i j}\right|+\left|p_{i j}\right|+\left|q_{i j}\right|\right. \\
& \left.+4 n \mu L_{i}^{g} h_{i j}\left(d_{i j}^{2}+p_{i j}^{2}+q_{i j}^{2}\right)\right) \\
& \times \int_{0}^{\infty} e_{p}(t+s, 0) h_{i j}(s) u_{i}^{2}(t) \Delta s \\
& -\sum_{j=1}^{m} \sum_{i=1}^{n} L_{i}^{g}(1+p \mu) \\
& \left.\left.\times\left(\left|c_{j i}\right|+\left|\alpha_{j i}\right|+\left|\beta_{j i}\right|\right)\right]\right\} \\
& \times u_{i}^{2}(t)+e_{p}(t, 0) \sum_{i=1}^{n} \sum_{j=1}^{m}(1+p \mu) \\
& \times\left\{\left[L_{j}^{f}\left(\left|c_{j i}\right|+\left|\alpha_{j i}\right|+\left|\beta_{j i}\right|\right)\right.\right. \\
& \times\left(\left|d_{i j}\right|+\left|p_{i j}\right|+\left|q_{i j}\right|\right. \\
& \left.+4 m \mu\left(L_{j}^{f}\right)^{2} k_{j i}\left(c_{j i}^{2}+\alpha_{j i}^{2}+\beta_{j i}^{2}\right)\right] \\
& \left.+4 n \mu L_{i}^{g} h_{i j}\left(d_{i j}^{2}+p_{i j}^{2}+q_{i j}^{2}\right)\right) \\
& \times \int_{0}^{\infty} e_{p}(t, 0) h_{i j}(s) u_{i}^{2}(t-s) \Delta s \\
& \left.\times \int_{0}^{\infty} k_{j i}(s) v_{j}^{2}(t-s) \Delta s\right\} \\
& +\sum_{j=1}^{m} e_{p}(t, 0) \\
& =\sum_{j=1}^{m} \sum_{i=1}^{n} L_{i}^{g}(1+p \mu) \\
& \times\left(\left|d_{i j}\right|+\left|p_{i j}\right|+\left|q_{i j}\right|\right. \\
& \times\{p+(1+p \mu) \\
& \left.+4 n \mu L_{i}^{g} h_{i j}\left(d_{i j}^{2}+p_{i j}^{2}+q_{i j}^{2}\right)\right) \\
& \times e_{p}(t, 0) \int_{0}^{\infty} e_{p}(t+s, t) h_{i j}(s) \Delta s u_{i}^{2}(t) \\
& \times\left[4 b_{j}^{2} \mu-2 b_{j}\right. \\
& -e_{p}(t, 0)(1+p \mu) \\
& \left.\left.+\sum_{i=1}^{n} L_{i}^{g} h_{i j}\left(\left|d_{i j}\right|+\left|p_{i j}\right|+\left|q_{i j}\right|\right)\right]\right\} v_{j}^{2}(t) \\
& \times \sum_{j=1}^{m} \sum_{i=1}^{n} L_{i}^{g}\left(\left|d_{i j}\right|+\left|p_{i j}\right|+\left|q_{i j}\right|\right. \\
& \left.+4 n \mu L_{i}^{g} h_{i j}\left(d_{i j}^{2}+p_{i j}^{2}+q_{i j}^{2}\right)\right) \\
& \times \int_{0}^{\infty} h_{j i}(s) u_{i}^{2}(t-s) \Delta s .
\end{aligned}
$$




$$
\begin{aligned}
& \times \int_{0}^{\infty} h_{i j}(s) u_{i}^{2}(t-s) \Delta s \\
& +\sum_{i=1}^{n} \sum_{j=1}^{m} L_{j}^{f}(1+p \mu) \\
& \times\left(\left|c_{j i}\right|+\left|\alpha_{j i}\right|+\left|\beta_{j i}\right|\right. \\
& \left.+4 m \mu L_{j}^{f} k_{j i}\left(c_{j i}^{2}+\alpha_{j i}^{2}+\beta_{j i}^{2}\right)\right) e_{p}(t, 0) \\
& \times \int_{0}^{\infty} e_{p}(t+s, t) k_{j i}(s) \Delta s v_{j}^{2}(t)-e_{p}(t, 0)(1+p \mu) \\
& \times \sum_{i=1}^{n} \sum_{j=1}^{m} L_{j}^{f}\left(\left|c_{j i}\right|+\left|\alpha_{j i}\right|+\left|\beta_{j i}\right|\right. \\
& \left.+4 m L_{j}^{f} k_{j i}\left(c_{j i}^{2}+\alpha_{j i}^{2}+\beta_{j i}^{2}\right)\right) \\
& \times \int_{0}^{\infty} k_{j i}(s) v_{j}^{2}(t-s) \Delta s \\
& +\sum_{j=1}^{m} \sum_{i=1}^{n} L_{i}^{g}(1+p \mu)\left(\left|d_{i j}\right|+\left|p_{i j}\right|+\left|q_{i j}\right|\right. \\
& \left.+4 n \mu L_{i}^{g} h_{i j}\left(d_{i j}^{2}+p_{i j}^{2}+q_{i j}^{2}\right)\right) \\
& \times e_{p}(t, 0) \int_{0}^{\infty} e_{p}(t+s, t) h_{i j}(s) \Delta s u_{i}^{2}(t) \\
& -e_{p}(t, 0)(1+p \mu) \\
& \times \sum_{j=1}^{m} \sum_{i=1}^{n} L_{i}^{g}\left(\left|d_{i j}\right|+\left|p_{i j}\right|+\left|q_{i j}\right|\right. \\
& \left.+4 n \mu L_{i}^{g} h_{i j}\left(d_{i j}^{2}+p_{i j}^{2}+q_{i j}^{2}\right)\right) \\
& \times \int_{0}^{\infty} h_{j i}(s) u_{i}^{2}(t-s) \Delta s \\
& \leq \sum_{i=1}^{n} e_{p}(t, 0)\{[p+(1+p \mu) \\
& \times\left(4 a_{i}^{2} \mu-2 a_{i}\right. \\
& +\sum_{j=1}^{m} L_{j}^{f} k_{j i} \\
& \left.\left.\times\left(\left|c_{j i}\right|+\left|\alpha_{j i}\right|+\left|\beta_{j i}\right|\right)\right)\right] \\
& +\sum_{j=1}^{m} L_{i}^{g}(1+p \mu) \\
& \times\left(\left|d_{i j}\right|+\left|p_{i j}\right|+\left|q_{i j}\right|\right. \\
& \left.+4 n \mu L_{i}^{g} h_{i j}\left(d_{i j}^{2}+p_{i j}^{2}+q_{i j}^{2}\right)\right)
\end{aligned}
$$$$
\left.\times \int_{0}^{\infty} e_{p}(t+s, t) h_{i j}(s) \Delta s\right\} u_{i}^{2}(t)
$$$$
+\sum_{j=1}^{m} e_{p}(t, 0)\{[p+(1+p \mu)
$$$$
\times\left(4 b_{j}^{2} \mu-2 b_{j}\right.
$$$$
\left.\left.+\sum_{i=1}^{n} L_{i}^{g} h_{i j}\left(\left|d_{i j}\right|+\left|p_{i j}\right|+\left|q_{i j}\right|\right)\right)\right]
$$$$
+\sum_{i=1}^{n} L_{j}^{f}(1+p \mu)
$$$$
\times\left(\left|c_{j i}\right|+\left|\alpha_{j i}\right|+\left|\beta_{j i}\right|\right.
$$$$
+4 m \mu L_{j}^{f}
$$$$
\left.\times k_{j i}\left(c_{j i}^{2}+\alpha_{j i}^{2}+\beta_{j i}^{2}\right)\right)
$$$$
\left.\times \int_{0}^{\infty} e_{p}(t+s, t) k_{j i}(s) \Delta s\right\} v_{j}^{2}(t) .
$$

By $\left(H_{4}\right)$, we can conclude that $V^{\Delta}(t) \leq 0$, for $t \in \mathbb{T}^{+}, t \neq t_{k}$, $k=1,2, \ldots$, which implies that $V(t) \leq V(0)$, for $t \in \mathbb{T}^{+}, t \neq t_{k}$, $k=1,2, \ldots$. For $t=t_{k}, k=1,2, \ldots$, we have

$$
\begin{aligned}
V\left(t_{k}^{+}\right)= & V_{1}\left(t_{k}^{+}\right)+V_{2}\left(t_{k}^{+}\right)+V_{3}\left(t_{k}^{+}\right)+V_{4}\left(t_{k}^{+}\right) \\
= & \sum_{i=1}^{n} u_{i}^{2}\left(t_{k}^{+}\right) e_{p}\left(t_{k}^{+}, 0\right)+\sum_{j=1}^{m} v_{j}^{2}\left(t_{k}^{+}\right) e_{p}\left(t_{k}^{+}, 0\right) \\
& +\sum_{i=1}^{n} \sum_{j=1}^{m} L_{j}^{f}(1+p \mu) \\
& \times\left(\left|c_{j i}\right|+\left|\alpha_{j i}\right|+\left|\beta_{j i}\right|\right. \\
& \left.+4 m \mu L_{j}^{f} k_{j i}\left(c_{j i}^{2}+\alpha_{j i}^{2}+\beta_{j i}^{2}\right)\right) \\
& \times \int_{0}^{\infty} \int_{t_{k}^{+}-s}^{t_{k}^{+}} e_{p}(r+s, 0) k_{j i}(s) v_{j}^{2}(r) \Delta r \Delta s \\
& +\sum_{j=1}^{m} \sum_{i=1}^{n} L_{i}^{g}(1+p \mu) \\
& \times\left(\left|d_{i j}\right|+\left|p_{i j}\right|+\left|q_{i j}\right|\right. \\
& \left.+4 n \mu L_{i}^{g} h_{i j}\left(d_{i j}^{2}+p_{i j}^{2}+q_{i j}^{2}\right)\right) \\
& \times \int_{0}^{\infty} \int_{t_{k}^{+}-s}^{t_{k}^{+}} e_{p}(r+s, 0) h_{i j}(s) u_{i}^{2}(r) \Delta r \Delta s
\end{aligned}
$$


Journal of Applied Mathematics

13

$$
\begin{aligned}
& \leq \sum_{i=1}^{n}\left(\left(1-\gamma_{i k}\right) u_{i}\left(t_{k}\right)\right)^{2} e_{p}\left(t_{k}, 0\right) \\
& +\sum_{j=1}^{m}\left(\left(1-\bar{\gamma}_{j k}\right) v_{j}\left(t_{k}\right)\right)^{2} e_{p}\left(t_{k}, 0\right) \\
& +\sum_{i=1}^{n} \sum_{j=1}^{m} L_{j}^{f}(1+p \mu) \\
& \times\left(\left|c_{j i}\right|+\left|\alpha_{j i}\right|+\left|\beta_{j i}\right|\right. \\
& \left.+4 m \mu L_{j}^{f} k_{j i}\left(c_{j i}^{2}+\alpha_{j i}^{2}+\beta_{j i}^{2}\right)\right) \\
& \times \int_{0}^{\infty} \int_{t_{k}-s}^{t_{k}} e_{p}(r+s, 0) k_{j i}(s) v_{j}^{2}(r) \Delta r \Delta s \\
& +\sum_{j=1}^{m} \sum_{i=1}^{n} L_{i}^{g}(1+p \mu) \\
& \times\left(\left|d_{i j}\right|+\left|p_{i j}\right|+\left|q_{i j}\right|\right. \\
& \left.+4 n \mu L_{i}^{g} h_{i j}\left(d_{i j}^{2}+p_{i j}^{2}+q_{i j}^{2}\right)\right) \\
& \times \int_{0}^{\infty} \int_{t_{k}-s}^{t_{k}} e_{p}(r+s, 0) h_{i j}(s) u_{i}^{2}(r) \Delta r \Delta s \\
& \leq \sum_{i=1}^{n} u_{i}^{2}\left(t_{k}\right) e_{p}\left(t_{k}, 0\right)+\sum_{j=1}^{m} v_{j}^{2}\left(t_{k}\right) e_{p}\left(t_{k}, 0\right) \\
& +\sum_{i=1}^{n} \sum_{j=1}^{m} L_{j}^{f}(1+p \mu)\left(\left|c_{j i}\right|+\left|\alpha_{j i}\right|+\left|\beta_{j i}\right|\right. \\
& \left.+4 m \mu L_{j}^{f} k_{j i}\left(c_{j i}^{2}+\alpha_{j i}^{2}+\beta_{j i}^{2}\right)\right) \\
& \times \int_{0}^{\infty} \int_{t_{k}-s}^{t_{k}} e_{p}(r+s, 0) k_{j i}(s) v_{j}^{2}(r) \Delta r \Delta s \\
& +\sum_{j=1}^{m} \sum_{i=1}^{n} L_{i}^{g}(1+p \mu)\left(\left|d_{i j}\right|+\left|p_{i j}\right|+\left|q_{i j}\right|\right. \\
& \left.+4 n \mu L_{i}^{g} h_{i j}\left(d_{i j}^{2}+p_{i j}^{2}+q_{i j}^{2}\right)\right) \\
& \times \int_{0}^{\infty} \int_{t_{k}-s}^{t_{k}} e_{p}(r+s, 0) h_{i j}(s) u_{i}^{2}(r) \Delta r \Delta s \\
& =V\left(t_{k}\right) \text {. }
\end{aligned}
$$

So we get $V(t) \leq V(0)$, for all $t \in \mathbb{T}^{+}$. Now, we estimate the value of $V(0)$. We have that

$$
\begin{aligned}
V_{1}(0) & =\sum_{i=1}^{n} u_{i}^{2}(0) e_{p}(0,0)=\sum_{i=1}^{n} u_{i}^{2}(0) \\
& \leq \sum_{i=1}^{n} \sup _{t \in(-\infty, 0]_{\mathbb{T}}} u_{i}^{2}(t),
\end{aligned}
$$

$$
\begin{aligned}
& V_{2}(0)=\sum_{j=1}^{m} v_{j}^{2}(0) e_{p}(0,0)=\sum_{j=1}^{m} v_{j}^{2}(0) \\
& \leq \sum_{j=1}^{m} \sup _{t \in(-\infty, 0]_{\mathbb{T}}} v_{j}^{2}(t), \\
& V_{3}(0)=\sum_{i=1}^{n} \sum_{j=1}^{m} L_{j}^{f}(1+p \mu) \\
& \times\left(\left|c_{j i}\right|+\left|\alpha_{j i}\right|+\left|\beta_{j i}\right|\right. \\
& \left.+4 m \mu L_{j}^{f} k_{j i}\left(c_{j i}^{2}+\alpha_{j i}^{2}+\beta_{j i}^{2}\right)\right) \\
& \times \int_{0}^{\infty} \int_{-s}^{0} e_{p}(r+s, 0) k_{j i}(s) v_{j}^{2}(r) \Delta r \Delta s \\
& \leq \sum_{i=1}^{n} \sum_{j=1}^{m} L_{j}^{f}(1+p \mu) \\
& \times\left(\left|c_{j i}\right|+\left|\alpha_{j i}\right|+\left|\beta_{j i}\right|\right. \\
& \left.+4 m \mu L_{j}^{f} k_{j i}\left(c_{j i}^{2}+\alpha_{j i}^{2}+\beta_{j i}^{2}\right)\right) \\
& \times \int_{0}^{\infty} \int_{-s}^{0} e_{p}(r+s, 0) k_{j i}(s) \Delta r \Delta s \\
& \times \sup _{t \in(-\infty, 0]_{\pi}} v_{j}^{2}(t) \\
& \leq \max _{1 \leq j \leq m}\left\{\sum_{i=1}^{n} L_{j}^{f}(1+p \mu)\right. \\
& \times\left(\left|c_{j i}\right|+\left|\alpha_{j i}\right|+\left|\beta_{j i}\right|\right. \\
& \left.+4 m \mu L_{j}^{f} k_{j i}\left(c_{j i}^{2}+\alpha_{j i}^{2}+\beta_{j i}^{2}\right)\right) \\
& \left.\times \int_{0}^{\infty} \int_{-s}^{0} e_{p}(r+s, 0) k_{j i}(s) \Delta r \Delta s\right\} \\
& \times \sum_{j=1}^{m} \sup _{t \in(-\infty, 0]_{\pi}} v_{j}^{2}(t), \\
& V_{4}(0)=\sum_{j=1}^{m} \sum_{i=1}^{n} L_{i}^{g}(1+p \mu) \\
& \times\left(\left|d_{i j}\right|+\left|p_{i j}\right|+\left|q_{i j}\right|\right. \\
& \left.+4 n \mu L_{i}^{g} h_{i j}\left(d_{i j}^{2}+p_{i j}^{2}+q_{i j}^{2}\right)\right) \\
& \times \int_{0}^{\infty} \int_{-s}^{0} e_{p}(r+s, 0) h_{i j}(s) u_{i}^{2}(r) \Delta r \Delta s \\
& \leq \sum_{j=1}^{m} \sum_{i=1}^{n} L_{i}^{g}(1+p \mu) \\
& \times\left(\left|d_{i j}\right|+\left|p_{i j}\right|+\left|q_{i j}\right|\right. \\
& \left.+4 n \mu L_{i}^{g} h_{i j}\left(d_{i j}^{2}+p_{i j}^{2}+q_{i j}^{2}\right)\right)
\end{aligned}
$$




$$
\begin{gathered}
\times \int_{0}^{\infty} \int_{-s}^{0} e_{p}(r+s, 0) h_{i j}(s) \Delta r \Delta s \\
\times \sup _{t \in(-\infty, 0]_{\top}} u_{i}^{2}(t) \\
\leq \max _{1 \leq i \leq n}\left\{\sum_{j=1}^{m} \sum_{i=1}^{n} L_{i}^{g}(1+p \mu)\right. \\
\times\left(\left|d_{i j}\right|+\left|p_{i j}\right|+\left|q_{i j}\right|\right. \\
\left.\quad+4 n \mu L_{i}^{g} h_{i j}\left(d_{i j}^{2}+p_{i j}^{2}+q_{i j}^{2}\right)\right) \\
\left.\times \int_{0}^{\infty} \int_{-s}^{0} e_{p}(r+s, 0) h_{j i}(s) \Delta r \Delta s\right\} \\
\times \sum_{i=1}^{n} \sup _{t \in(-\infty, 0]_{\pi}} u_{i}^{2}(t) .
\end{gathered}
$$

It follows that

$$
\begin{aligned}
& V(0) \leq \max _{1 \leq i \leq n}\left\{1+\sum_{j=1}^{m} \sum_{i=1}^{n} L_{i}^{g}(1+p \mu)\right. \\
& \times\left(\left|d_{i j}\right|+\left|p_{i j}\right|+\left|q_{i j}\right|\right. \\
& \left.+4 n \mu L_{i}^{g} h_{i j}\left(d_{i j}^{2}+p_{i j}^{2}+q_{i j}^{2}\right)\right) \\
& \left.\times \int_{0}^{\infty} \int_{-s}^{0} e_{p}(r+s, 0) h_{j i}(s) \Delta r \Delta s\right\} \\
& \times \sum_{i=1}^{n} \sup _{t \in(-\infty, 0]_{\pi}} u_{i}^{2}(t) \\
& +\max _{1 \leq j \leq m}\left\{1+\sum_{i=1}^{n} L_{j}^{f}(1+p \mu)\right. \\
& \times\left(\left|c_{j i}\right|+\left|\alpha_{j i}\right|+\left|\beta_{j i}\right|\right. \\
& \left.+4 m \mu L_{j}^{f} k_{j i}\left(c_{j i}^{2}+\alpha_{j i}^{2}+\beta_{j i}^{2}\right)\right) \\
& \left.\times \int_{0}^{\infty} \int_{-s}^{0} e_{p}(r+s, 0) k_{j i}(s) \Delta r \Delta s\right\} \\
& \times \sum_{j=1}^{m} \sup _{t \in(-\infty, 0]_{\top}} v_{j}^{2}(t) .
\end{aligned}
$$

Observe that

$$
\begin{aligned}
V(t) & \geq V_{1}(t)+V_{2}(t) \\
& \geq e_{p}(t, 0)\left(\sum_{i=1}^{n} u_{i}^{2}(t)+\sum_{j=1}^{m} v_{j}^{2}(t)\right) .
\end{aligned}
$$

So we have

$$
\begin{aligned}
& \sum_{i=1}^{n} u_{i}^{2}(t)+\sum_{j=1}^{m} v_{j}^{2}(t) \\
& \leq M e_{\ominus p}(t, 0)\left(\sum_{i=1}^{n} \sup _{t \in(-\infty, 0]} u_{i}^{2}(t)+\sum_{j=1}^{m} \sup _{t \in(-\infty, 0]} v_{j}^{2}(t)\right), \\
& \forall t \in \mathbb{T}^{+},
\end{aligned}
$$

where

$$
\begin{aligned}
& M=\max \left\{\operatorname { m a x } _ { 1 \leq i \leq n } \left\{1+\sum_{j=1}^{m} \sum_{i=1}^{n} L_{i}^{g}(1+p \mu)\right.\right. \times\left(\left|d_{i j}\right|+\left|p_{i j}\right|+\left|q_{i j}\right|\right. \\
&\left.+4 n \mu L_{i}^{g} h_{i j}\left(d_{i j}^{2}+p_{i j}^{2}+q_{i j}^{2}\right)\right) \\
&\left.\times \int_{0}^{\infty} \int_{-s}^{0} e_{p}(r+s, 0) h_{j i}(s) \Delta r \Delta s\right\}, \\
& \max _{1 \leq j \leq m}\left\{1+\sum_{i=1}^{n} L_{j}^{f}(1+p \mu)\right. \\
& \times\left(\left|c_{j i}\right|+\left|\alpha_{j i}\right|+\left|\beta_{j i}\right|+4 m \mu L_{j}^{f} k_{j i}\right. \\
&\left.\left.\times \int_{0}^{\infty} \int_{-s}^{0} e_{p}(r+s, 0) k_{j i}(s) \Delta r \Delta s\right\}\right\} .
\end{aligned}
$$

It is obvious that $M>1$. Therefore, the equilibrium point $z^{*}$ of (28) is exponentially stable. This completes the proof of Theorem 19.

\section{An Example}

In this section, an example is given to verify the feasibility of our results obtained in previous sections.

Example 20. Let $n=m=2$. Consider the following fuzzy BAM neural networks without impulses on time scale $\mathbb{T}=\mathbb{R}$ or $\mathbb{Z}$ :

$$
\begin{aligned}
x_{i}^{\Delta}(t)= & -a_{i} x_{i}(t) \\
& +\sum_{j=1}^{2} c_{j i} \int_{0}^{\infty} k_{j i}(s) f_{j}\left(y_{j}(t-s)\right) \Delta s \\
& +\bigwedge_{j=1}^{2} \alpha_{j i} \int_{0}^{\infty} k_{j i}(s) f_{j}\left(y_{j}(t-s)\right) \Delta s
\end{aligned}
$$




$$
\begin{aligned}
& +\bigvee_{j=1}^{2} \beta_{j i} \int_{0}^{\infty} k_{j i}(s) f_{j}\left(y_{j}(t-s)\right) \Delta s \\
& +\bigwedge_{j=1}^{2} T_{j i} \mu_{j}+\bigvee_{j=1}^{2} H_{j i} \mu_{j}+I_{i}, \quad t \in \mathbb{T}^{+}, i=1,2, \\
y_{j}^{\Delta}(t)= & -b_{j} y_{j}(t) \\
& +\sum_{i=1}^{2} d_{i j} \int_{0}^{\infty} h_{i j}(s) g_{i}\left(x_{i}(t-s)\right) \Delta s \\
& +\bigwedge_{i=1}^{2} p_{i j} \int_{0}^{\infty} h_{i j}(s) g_{i}\left(x_{i}(t-s)\right) \Delta s \\
& +\bigvee_{i=1}^{2} q_{i j} \int_{0}^{\infty} h_{i j}(s) g_{i}\left(x_{i}(t-s)\right) \Delta s \\
& +\bigwedge_{i=1}^{2} F_{i j} v_{i}+\bigvee_{i=1}^{2} G_{i j} v_{i}+J_{j}, \quad t \in \mathbb{T}^{+}, j=1,2,
\end{aligned}
$$

where $\left(a_{1}, a_{2}\right)^{T}=(0.2,0.3)^{T},\left(b_{1}, b_{2}\right)^{T}=(0.2,0.5)^{T}$,

$$
\begin{gathered}
\left(\begin{array}{ll}
c_{11} & c_{12} \\
c_{21} & c_{22}
\end{array}\right)=\left(\begin{array}{ll}
\alpha_{11} & \alpha_{12} \\
\alpha_{21} & \alpha_{22}
\end{array}\right)=\left(\begin{array}{ll}
\beta_{11} & \beta_{12} \\
\beta_{21} & \beta_{22}
\end{array}\right)=\left(\begin{array}{cc}
0 & 0.01 \\
0.02 & 0
\end{array}\right), \\
\left(\begin{array}{ll}
d_{11} & d_{12} \\
d_{21} & d_{22}
\end{array}\right)=\left(\begin{array}{ll}
p_{11} & p_{12} \\
p_{21} & p_{22}
\end{array}\right)=\left(\begin{array}{ll}
q_{11} & q_{12} \\
q_{21} & q_{22}
\end{array}\right)=\left(\begin{array}{cc}
0 & 0.03 \\
0.01 & 0
\end{array}\right), \\
f_{1}(u)=f_{2}(u)=g_{1}(u)=g_{2}(u)=0.1|u|, \\
k_{j i}(s)=h_{i j}(s)=e_{2}(0, s), \quad i, j=1,2 .
\end{gathered}
$$

We can easily see that $L_{1}^{f}=L_{2}^{f}=L_{1}^{g}=L_{2}^{g}=0.1$. If $\mathbb{T}=\mathbb{R}$, then $\mu(t)=0$; if $\mathbb{T}=\mathbb{Z}$ then $\mu(t)=1$. Hence, we have

$$
\int_{0}^{\infty} e_{2}(0, s) \Delta s= \begin{cases}\frac{1}{2}, & \text { if } \mathbb{T}=\mathbb{R} \\ \frac{1}{3 \ln 3}, & \text { if } \mathbb{T}=\mathbb{Z}\end{cases}
$$

So, we can take $k_{j i}=h_{i j}=1, i, j=1,2$. We can obtain that $\theta=0.06<1$. Moreover, if $\mu(t)=0$, we can obtain that

$$
\begin{aligned}
-2 a_{1} & +\sum_{j=1}^{2} L_{j}^{f} k_{j 1}\left(\left|c_{j 1}\right|+\left|\alpha_{j 1}\right|+\left|\beta_{j 1}\right|\right) \\
& +\sum_{j=1}^{2} L_{1}^{g}\left(\left|d_{1 j}\right|+\left|p_{1 j}\right|+\left|q_{1 j}\right|+h_{1 j}\right) \\
= & -0.17<0,
\end{aligned}
$$

$$
\begin{aligned}
-2 a_{2} & +\sum_{j=1}^{2} L_{j}^{f} k_{j 2}\left(\left|c_{j 2}\right|+\left|\alpha_{j 2}\right|+\left|\beta_{j 2}\right|\right) \\
& +\sum_{j=1}^{2} L_{1}^{g}\left(\left|d_{2 j}\right|+\left|p_{2 j}\right|+\left|q_{2 j}\right|+h_{2 j}\right) \\
= & -0.37<0, \\
-2 b_{1} & +\sum_{i=1}^{2} L_{i}^{g} h_{i 1}\left(\left|d_{i 1}\right|+\left|p_{i 1}\right|+\left|q_{i 1}\right|\right) \\
& +\sum_{i=1}^{2} L_{j}^{f}\left(\left|c_{1 i}\right|+\left|\alpha_{1 i}\right|+\left|\beta_{1 i}\right|+k_{1 i}\right) \\
= & -0.17<0, \\
-2 b_{2} & +\sum_{i=1}^{2} L_{i}^{g} h_{i 2}\left(\left|d_{i 2}\right|+\left|p_{i 2}\right|+\left|q_{i 2}\right|\right) \\
& +\sum_{i=1}^{2} L_{j}^{f}\left(\left|c_{2 i}\right|+\left|\alpha_{2 i}\right|+\left|\beta_{2 i}\right|+k_{2 i}\right) \\
= & -0.77<0 .
\end{aligned}
$$

If $\mu(t)=1$, we have that

$$
\begin{aligned}
& 4 a_{1}^{2}-2 a_{1}+\sum_{j=1}^{2} L_{j}^{f} k_{j 1}\left(\left|c_{j 1}\right|+\left|\alpha_{j 1}\right|+\left|\beta_{j 1}\right|\right) \\
& +\sum_{j=1}^{2} L_{1}^{g} h_{1 j}\left(\left|d_{1 j}\right|+\left|p_{1 j}\right|+\left|q_{1 j}\right|\right. \\
& \left.+4 n L_{1}^{g}\left(d_{1 j}^{2}+p_{1 j}^{2}+q_{1 j}^{2}\right)+h_{1 j}\right) \\
& \approx-0.0336<0, \\
& 4 a_{2}^{2}-2 a_{2}+\sum_{j=1}^{2} L_{j}^{f} k_{j 2}\left(\left|c_{j 2}\right|+\left|\alpha_{j 2}\right|+\left|\beta_{j 2}\right|\right) \\
& +\sum_{j=1}^{2} L_{2}^{g} h_{2 j}\left(\left|d_{2 j}\right|+\left|p_{2 j}\right|+\left|q_{2 j}\right|\right. \\
& \left.\quad+4 n L_{2}^{g}\left(d_{2 j}^{2}+p_{2 j}^{2}+q_{2 j}^{2}\right)+h_{2 j}\right) \\
& \approx-0.0374<0, \\
& 4 b_{1}^{2}-2 b_{1}+\sum_{i=1}^{2} L_{i}^{g} h_{i 1}\left(\left|d_{i 1}\right|+\left|p_{i 1}\right|+\left|q_{i 1}\right|\right) \\
& +\sum_{i=1}^{2} L_{1}^{f} k_{1 i}\left(\left|c_{1 i}\right|+\left|\alpha_{1 i}\right|+\left|\beta_{1 i}\right|\right. \\
& \approx-0.0336<0, \\
& \left.+4 m L_{1}^{f} k_{1 i}\left(c_{1 i}^{2}+\alpha_{1 i}^{2}+\beta_{1 i}^{2}\right)+k_{1 i}\right)
\end{aligned}
$$




$$
\begin{aligned}
& 4 b_{2}^{2}-2 b_{2}+\sum_{i=1}^{2} L_{i}^{g} h_{i 12}\left(\left|d_{i 2}\right|+\left|p_{i 2}\right|+\left|q_{i 2}\right|\right) \\
& +\sum_{i=1}^{2} L_{2}^{f} k_{2 i}\left(\left|c_{2 i}\right|+\left|\alpha_{2 i}\right|+\left|\beta_{2 i}\right|\right. \\
& \left.+4 m L_{2}^{f} k_{2 i}\left(c_{2 i}^{2}+\alpha_{2 i}^{2}+\beta_{2 i}^{2}\right)+k_{2 i}\right) \\
& \approx-0.0381<0
\end{aligned}
$$

which imply that all assumptions in Theorems 18 and 19 are satisfied. Thus, it follows from Theorems 18 and 19 that (42) has one unique equilibrium point, which is exponentially stable.

Remark 21. The conclusion of Example 1 cannot be obtained by the results obtained in [22, 30-32].

\section{Conclusion}

In this paper, using the time scale calculus theory, the fixed point theory, and Lyapunov functional method, some sufficient conditions are obtained to ensure the existence and the exponential stability of unique equilibrium point of a class of fuzzy BAM neural networks with continuously distributed delays and impulses on time scales. Since it is troublesome to study the existence and stability of equilibrium points for continuous and discrete delay systems, respectively, it is significant to study that on time scales which can unify the continuous and discrete time situations. The sufficient conditions we obtained can easily be checked in practice by simple algebraic methods.

\section{Conflict of Interests}

The authors declare that there is no conflict of interests regarding the publication of this paper.

\section{Acknowledgment}

This work is supported by the National Natural Sciences Foundation of China under Grant no. 11361072.

\section{References}

[1] B. Kosko, "Bidirectional associative memories," IEEE Transactions on Systems, Man, and Cybernetics, vol. 18, no. 1, pp. 49-60, 1988.

[2] Y. Li, "Existence and stability of periodic solution for BAM neural networks with distributed delays," Applied Mathematics and Computation, vol. 159, no. 3, pp. 847-862, 2004.

[3] Q. Zhou, "Global exponential stability of BAM neural networks with distributed delays and impulses," Nonlinear Analysis: Real World Applications, vol. 10, no. 1, pp. 144-153, 2009.

[4] B. Zheng, Y. Zhang, and C. Zhang, "Global existence of periodic solutions on a simplified BAM neural network model with delays," Chaos, Solitons \& Fractals, vol. 37, no. 5, pp. 1397-1408, 2008.
[5] Y. Xia, J. Cao, and M. Lin, "New results on the existence and uniqueness of almost periodic solution for BAM neural networks with continuously distributed delays," Chaos, Solitons \& Fractals, vol. 31, no. 4, pp. 928-936, 2007.

[6] L. Zhang and L. Si, "Existence and exponential stability of almost periodic solution for BAM neural networks with variable coefficients and delays," Applied Mathematics and Computation, vol. 194, no. 1, pp. 215-223, 2007.

[7] C. Liu, C. Li, and X. Liao, "Variable-time impulses in BAM neural networks with delays," Neurocomputing, vol. 74, no. 17, pp. 3286-3295, 2011.

[8] Y. Li, "Global exponential stability of BAM neural networks with delays and impulses," Chaos, Solitons \& Fractals, vol. 24, no. 1, pp. 279-285, 2005.

[9] Y. Li and X. Fan, "Existence and globally exponential stability of almost periodic solution for Cohen-Grossberg BAM neural networks with variable coefficients," Applied Mathematical Modelling, vol. 33, no. 4, pp. 2114-2120, 2009.

[10] J. H. Park, C. H. Park, O. M. Kwon, and S. M. Lee, "A new stability criterion for bidirectional associative memory neural networks of neutral-type," Applied Mathematics and Computation, vol. 199, no. 2, pp. 716-722, 2008.

[11] J. Liu and G. Zong, "New delay-dependent asymptotic stability conditions concerning BAM neural networks of neutral type," Neurocomputing, vol. 72, no. 10-12, pp. 2549-2555, 2009.

[12] T. Yang, L.-B. Yang, C. W. Wu, and L. O. Chua, "Fuzzy cellular neural networks: theory," in Proceedings of the 4th IEEE International Workshop on Cellular Neural Networks, and Their Applications (CNNA '96), pp. 181-186, June 1996.

[13] T. Huang, "Exponential stability of fuzzy cellular neural networks with distributed delay," Physics Letters A, vol. 351, no. 1-2, pp. 48-52, 2006.

[14] K. Yuan, J. Cao, and J. Deng, "Exponential stability and periodic solutions of fuzzy cellular neural networks with time-varying delays," Neurocomputing, vol. 69, no. 13-15, pp. 1619-1627, 2006.

[15] Q. Zhang and R. Xiang, "Global asymptotic stability of fuzzy cellular neural networks with time-varying delays," Physics Letters A, vol. 372, no. 22, pp. 3971-3977, 2008.

[16] Q. Song and J. Cao, "Dynamical behaviors of discrete-time fuzzy cellular neural networks with variable delays and impulses," Journal of the Franklin Institute, vol. 345, no. 1, pp. 39-59, 2008.

[17] J. Wang and J. G. Lu, "Global exponential stability of fuzzy cellular neural networks with delays and reaction-diffusion terms," Chaos, Solitons \& Fractals, vol. 38, no. 3, pp. 878-885, 2008.

[18] Y. Li, L. Yang, and W. Wu, "Periodic solutions for a class of fuzzy BAM neural networks with distributed delays and variable coefficients," International Journal of Bifurcation and Chaos in Applied Sciences and Engineering, vol. 20, no. 5, pp. 1551-1565, 2010.

[19] Y. Liu and W. Tang, "Exponential stability of fuzzy cellular neural networks with constant and time-varying delays," Physics Letters A, vol. 323, no. 3-4, pp. 224-233, 2004.

[20] C. Li, Y. Li, and Y. Ye, "Exponential stability of fuzzy CohenGrossberg neural networks with time delays and impulsive effects," Communications in Nonlinear Science and Numerical Simulation, vol. 15, no. 11, pp. 3599-3606, 2010.

[21] Y. Li and C. Wang, "Existence and global exponential stability of equilibrium for discrete-time fuzzy BAM neural networks with variable delays and impulses," Fuzzy Sets and Systems, vol. 217, pp. 62-79, 2013. 
[22] T. Huang, C. Li, and Z. Zeng, "A domain attraction criterion for interval fuzzy neural networks," Computers \& Mathematics with Applications, vol. 58, no. 3, pp. 508-513, 2009.

[23] Z. Zhang and K. Liu, "Existence and global exponential stability of a periodic solution to interval general Bidirectional Associative Memory (BAM) neural networks with multiple delays on time scales," Neural Networks, vol. 24, no. 5, pp. 427-439, 2011.

[24] Y. Li, X. Chen, and L. Zhao, "Stability and existence of periodic solutions to delayed Cohen-Grossberg BAM neural networks with impulses on time scales," Neurocomputing, vol. 72, no. 7-9, pp. 1621-1630, 2009.

[25] Y. Li and S. Gao, "Global exponential stability for impulsive BAM neural networks with distributed delays on time scales," Neural Processing Letters, vol. 31, no. 1, pp. 65-91, 2010.

[26] Y. Li and K. Zhao, "Robust stability of delayed reaction-diffusion recurrent neural networks with Dirichlet boundary conditions on time scales," Neurocomputing, vol. 74, no. 10, pp. 1632-1637, 2011.

[27] Y. K. Li, K. H. Zhao, and Y. Ye, "Stability of reaction-diffusion recurrent neural networks with distributed delays and neumann boundary conditions on time scales," Neural Processing Letters, vol. 36, no. 3, pp. 217-234, 2012.

[28] Y. Li, L. Yang, and W. Wu, "Anti-periodic solutions for a class of Cohen-Grossberg neutral networks with time-varying delays on time scales," International Journal of Systems Science, vol. 42, no. 7, pp. 1127-1132, 2011.

[29] B. Zhou, Q. Song, and H. Wang, "Global exponential stability of neural networks with discrete and distributed delays and general activation functions on time scales," Neurocomputing, vol. 74, no. 17, pp. 3142-3150, 2011.

[30] Y. K. Li, L. Yang, and L. J. Sun, "Existence and exponential stability of an equilibrium point for fuzzy BAM neural networks with time-varying delays in leakage terms on time scales," Advances in Difference Equations, vol. 2013, article 218, 2013.

[31] Y. Li and T. Zhang, "Global exponential stability of fuzzy interval delayed neural networks with impulses on time scales," International Journal of Neural Systems, vol. 19, no. 6, pp. 449456, 2009.

[32] Q. H. Zhang, L. H. Yang, and D. X. Liao, "Existence and globally exponential stability of equilibrium for fuzzy BAM neural networks with distributed delays and impulse," Advances in Difference Equations, vol. 2011, article 8, 2011.

[33] M. Bohner and A. Peterson, Dynamic Equations on Time Scales, Birkhäauser, Boston, Mass, USA, 2001. 


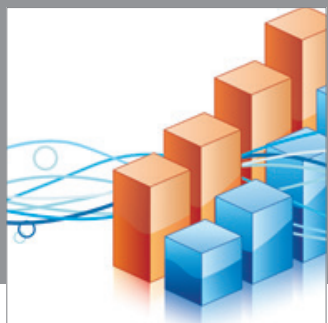

Advances in

Operations Research

mansans

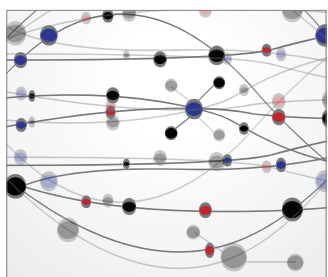

The Scientific World Journal
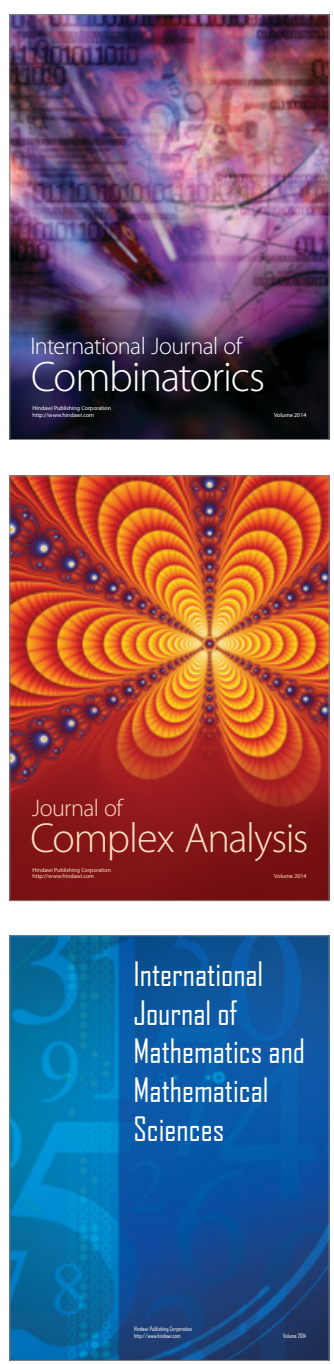
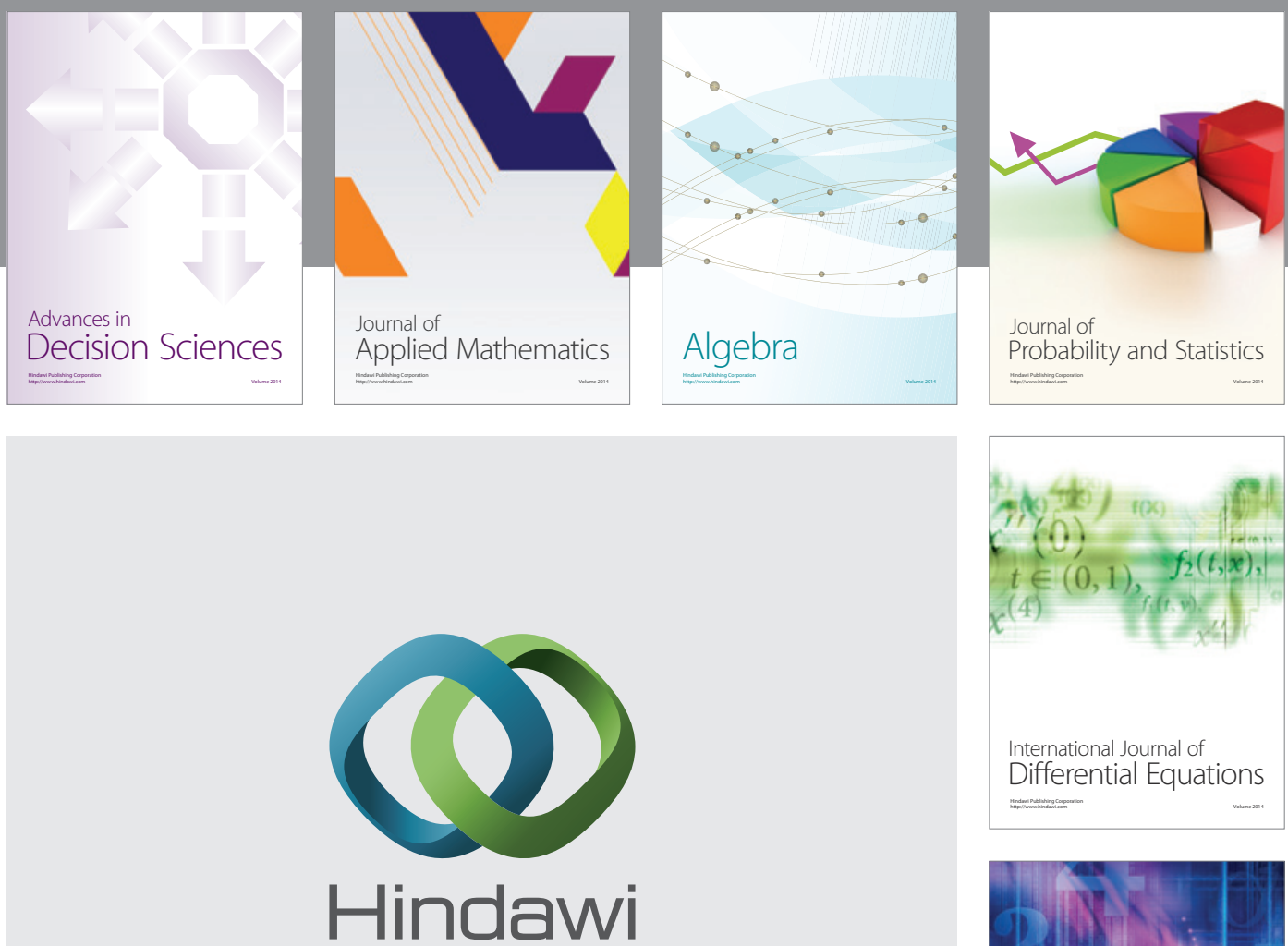

Submit your manuscripts at http://www.hindawi.com
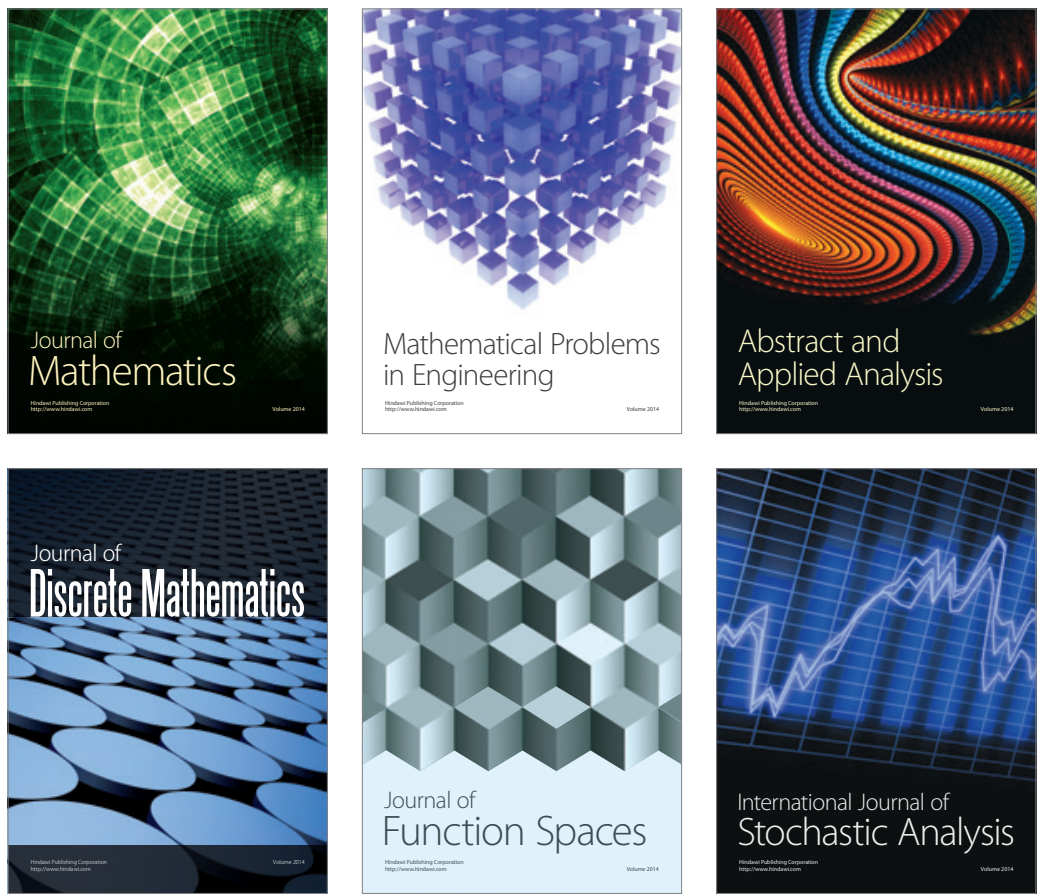

Journal of

Function Spaces

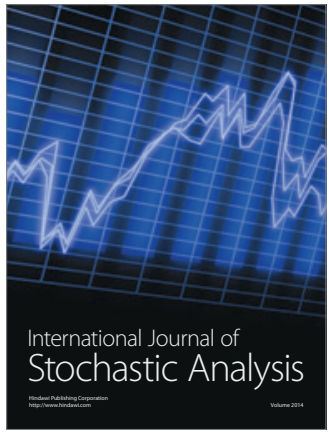

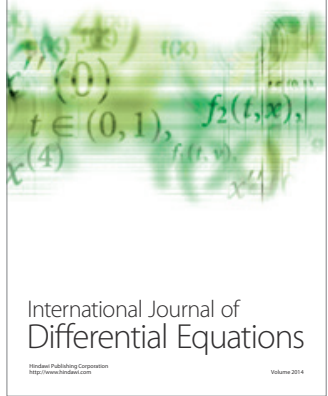
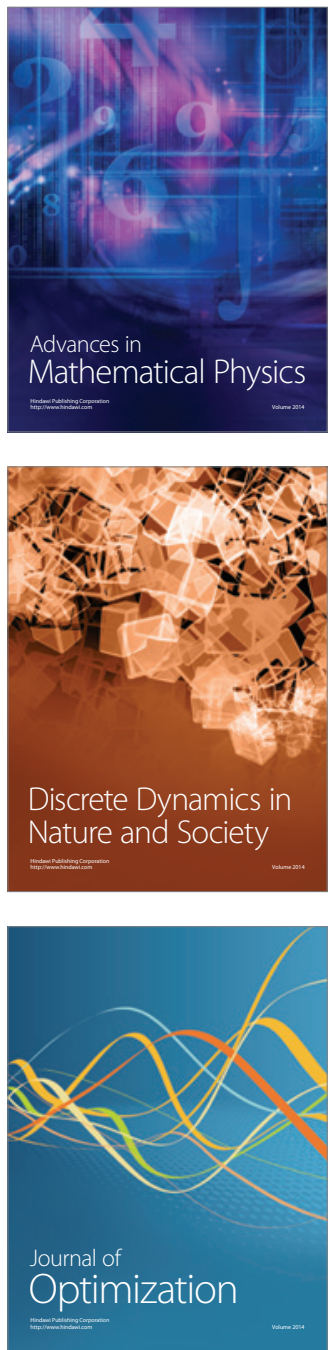